\title{
聚合物辅助设计生长高质量多功能薄膜
}

\author{
易庆华 ${ }^{1}$ 赵 杰 ${ }^{1}$ 娄艳辉 ${ }^{1}$ 邹贵付 ${ }^{1, *} \quad$ 刘忠范 $^{2}$ \\ ('苏州大学物理与光电·能源学部, 苏州纳米科技协同创新中心, 江苏苏州 215006 ; \\ ${ }^{2}$ 北京大学化学与分子工程学院, 北京 100871)
}

\begin{abstract}
摘要: 随着薄膜材料的日益发展和新型薄膜材料的不断涌现, 开发薄膜生长技术对于半导体和光电等科技领 域的作用日益突出。本文主要介绍最近发展的聚合物辅助沉积从分子层面上控制生长高质量的薄膜材料。聚 合物辅助沉积是一种生长高质量薄膜的化学水性溶液方法, 将金属离子与聚合物通过络合、氢键或静电等方 式形成一种均匀稳定的前驱体溶液, 再经过超滤、成膜和热处理形成高质量的金属氧化物、金属碳化物、金 属氮化物、金属单质、金属硫/硒化物等薄膜以及纳米粒子等化合物或复合功能材料。该方法中水溶性的聚 合物能通过络合作用抑制金属离子的水解使得溶液稳定, 并能精确控制薄膜的组分从而形成高质量的薄膜。 该化学溶液方法的提出为科学技术领域提供了一种低成本和大面积制备薄膜的技术路线。本文最后总结和展 望了聚合物辅助沉积法未来的挑战和发展方向。
\end{abstract}

关键词: 聚合物; 薄膜; 半导体; 化学溶液法; 复合功能材料

中图分类号: 0649

\section{Design and Growth of High-Quality Multifunctional Thin Films by Polymer-Assisted Deposition}

\author{
YI Qing-Hua ${ }^{1} \quad$ ZHAO Jie ${ }^{1} \quad$ LOU Yan-Hui ${ }^{1} \quad$ ZOU Gui-Fu ${ }^{1, *} \quad$ LIU Zhong-Fan ${ }^{2}$ \\ ('College of Physics, Optoelectronics and Energy, Collaborative Innovation Center of Suzhou Nano Science and Technology, \\ Soochow University, Suzhou 215006, Jiangsu Province, P. R. China; ${ }^{2}$ College of Chemistry and Molecular Engineering, \\ Peking University, Beijing 100871, P. R. China)
}

\begin{abstract}
With the development of thin film technology, new methods to grow thin films is emerging. This review introduces one type of polymer-assisted deposition to grow thin films. Polymer-assisted deposition is one of the chemical solution ways to grow high-quality thin films. In this process, metal ions coordinate with a polymer by covalent bonding, hydrogen bonding, or static electricity to form a stable precursor. The controllable viscosity and homogeneous solution system ensure high-quality growth of thin films or nanoparticles. The diverse components of thin film range from a metal-oxides, metal-carbides, metal-nitrides, metal-sulfides/selenides to elementary substance (e.g. metals) even dopant composites. This method provides an alternative strategy to grow thin films. In addition, the prospects and challenges of the polymer-assisted deposition are discussed in the review as well.
\end{abstract}

Key Words: Polymer; Thin film; Semiconductor; Chemical solution method; Multifunctional material

\section{1 引言}

薄膜生长早已是科学和技术领域的一门独立
的技术 ${ }^{1}$ 。薄膜材料由于其质量、体积及性能方面 的特点使得其在科学和技术等领域都具有广泛的

Received: September 7, 2016; Revised: October 21, 2016; Published online: November 9, 2016.

*Corresponding author. Email: zouguifu@suda.edu.cn; Tel: +86-512-65228130.

The project was supported by the National Key Basic Research Program of China (973) (2015CB358600) and Excellent Young Scholar Fund from

National Natural Science Foundation of China (21422103).

国家重点基础研究发展规划项目(973) (2015CB358600)及国家自然科学基金优秀青年基金(21422103)资助

(C) Editorial office of Acta Physico-Chimica Sinica 
应用, 如发光二极管 ${ }^{2}$ 、微波设备 ${ }^{3}$ 、传感器 ${ }^{4}$ 、光 学滤镜 5 、燃气浴轮发动机 ${ }^{6}$ 、光电转换器件 ${ }^{7-10}$ 、 光电探测器 ${ }^{11}$ 、激光二极管 ${ }^{12}$ 、透明电极 ${ }^{13-15}$ 、磁性 存储器 ${ }^{16}$ 、铁电压电和热电器件 ${ }^{17}$ 、超导元件 ${ }^{18}$ 等。 尤其是上个世纪 70 年代以来, 薄膜技术得到了突 飞猛进的发展, 在科研和生活中取了丰硕的成果, 薄膜制备和薄膜生长技术已经是当今社会最活跃的 领域之一。如何生长具有不同形式、有特定的结构 和功能特性且能满足日益发展的科学技术要求的薄 膜材料已经是一项越来越重要的先进技术。

\section{2 薄膜的生长方法}

薄膜生长的方法主要分为物理方法和化学方法。 物理方法主要包括分子束外延 ${ }^{19}$ 、电子束蒸 发 ${ }^{20}$ 、热蒸发 ${ }^{21}$ 、溅射 ${ }^{22}$ 、脉冲激光沉积 ${ }^{23}$ 等方法。 物理方法可有效地控制薄膜厚度、薄膜纯度以及 物理性质等特性, 但是设备昂贵、成膜面积小等

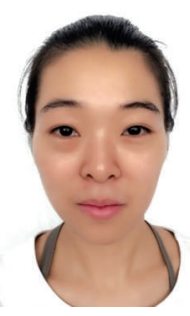

易庆华，2014年苏州大学材料物理 与化学专业硕士研究生, 现为苏州 大学新能源科学与工程专业全日制 非定向博士研究生。主要研究方向 为化学溶液法制备功能化合物薄膜。

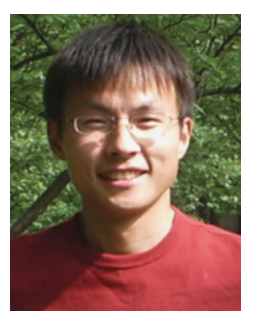

赵杰, 2012年获得苏州大学高分子 化学与物理专业博士学位, 现为苏 州大学能源学院副教授。主要研究 方向为功能离子液体、离子型聚合 物、功能薄膜材料、染料敏化等太 阳能电池。
因素限制了物理方法的广泛应用 ${ }^{24}$ 。另外物理方法 比较难实现在不规则的基底或者复杂的基底上沉 积薄膜。此外, 在金属氧化物薄膜生长过程中, 物理方法还需提供高氧压, 并且氧含量对功能氧 化物薄膜的性质具有至关重要的作用, 因此生长 环境会对薄膜的性能具有局限性 ${ }^{25,26}$ 。同时物理成 膜方法还包括溶液成膜方法, 如旋转涂敷法、喷 墨打印法等, 物理方法中的溶液成膜法是将所需 的材料制备成纳米颗粒, 再将纳米颗粒与溶剂、 粘结剂等物质混合得到 “墨水” 通过旋转涂敷或 喷墨打印等方式成膜, 这些方法成本低, 可以制 备出大面积的膜。但成膜后如果无后处理会引入 一些不必要的成份如粘结剂等。

化学方法设备相对简单, 成膜面积大, 可生 长多元和单元等功能复合薄膜, 是近年来科学研 究的热点, 但化学方法也有一定的局限性, 成膜 质量不如常规物理方法成膜质量高, 成份无法精 确控制等特点。常用的化学方法包括化学气相沉 积和化学溶液法两大类。化学气相沉积法通常是 在一个气相的氛围内气体之间的反应, 这个方法 在多种科学领域都有一定的应用, 而化学溶液法 成为了近年来科学技术领域中一种具有潜力的薄 膜制备方法。以下为几种常用的制备薄膜的化学 溶液法。

一般地, 溶胶凝胶法 ${ }^{27-29}$ 包括一个从液相的

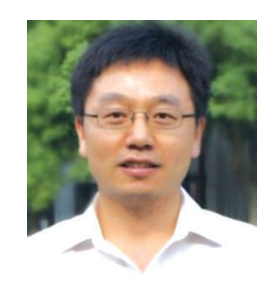

邹贵付, 江苏省特聘教授, 苏州大 学博士生导师, 国家青年 “973” 计划首席科学家, 国家优秀青年基 金和江苏省杰出青年基金获得者。 研究领域为无机微结构复合薄膜 及其光电应用基础研究, 主要集 中在功能薄膜生长与纳米结构复合、化学设计新 型太阳能电池结构及超导纳米线及其单光子检测 等方面。

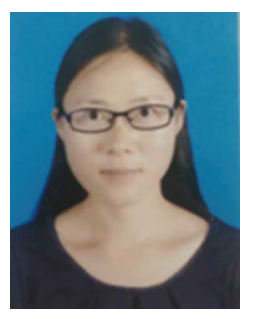

娄艳辉，2012年于日本富山大学获 得博士学位, 现为苏州大学能源学 院副教授。主要从事新型太阳能电 池的研究, 包括器件结构设计, 以 及物理机制方面的分析。

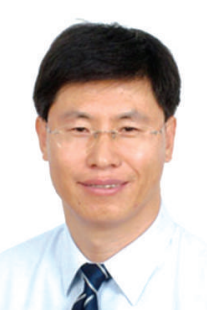

刘忠范, 中国科学院院士, 北京大 学化学与分子工程学院教授, 博士 生导师。主要从事纳米化学和纳米 结构器件研究, 发展了纳米碳材料 的化学气相沉积方法学, 建立了精 确调控碳纳米管、石墨烯等碳材料 结构的系列生长方法, 开拓了碳纳米管电子学的 材料与器件基础、基于扫描探针技术的超高密度 信息存储的研究方法。 
“溶胶” 到固相 “凝胶” 的过程, 是一种通过活泼 无机金属盐或者金属醇盐类的金属有机化合物作 为前驱体, 将前驱体均匀混合, 并进行水解、缩 合化学反应(如公式(1)、(2))形成透明溶胶体系, 溶胶经过陈化胶体聚合形成一定结构的凝胶或胶 体悬浮液, 将溶液或溶胶通过浸渍法或者旋涂成 膜法在基底上形成液膜, 再热处理使得凝胶分 解, 得到所需的薄膜。其中金属醇盐和无机金属 盐的水解度、 $\mathrm{pH}$ 值、溶液反应温度和络合剂等参 数对成膜质量起到了关键作用。

水解反应:

$\mathrm{M}(\mathrm{OR})_{n}+x \mathrm{H}_{2} \mathrm{O} \rightarrow \mathrm{M}(\mathrm{OH})_{x}(\mathrm{OR})_{n-x}+x \mathrm{ROH}$

聚合反应:

$-\mathrm{M}-\mathrm{OH}+\mathrm{HO}-\mathrm{M}-\rightarrow-\mathrm{M}-\mathrm{O}-\mathrm{M}-+$

$\mathrm{H}_{2} \mathrm{O}-\mathrm{M}-\mathrm{OR}+\mathrm{HO}-\mathrm{M}-\rightarrow$

$-\mathrm{M}-\mathrm{O}-\mathrm{M}-+\mathrm{ROH}$

化学浴的方法是一种历史悠久的薄膜生长方

法, 1884 年首次用硫脲来制备 $\mathrm{PbS}$ 薄膜的沉 积 ${ }^{30}$-32。这一方法主要是通过将基底浸泡在含有金 属盐和含硫化合物(硫䐂、硫代乙酰胺、硫代硫酸 盐、钠硫化物等)的溶液中来生长金属硫化物和金 属硒化物。该方法中金属离子的浓度和硫的浓度 必须高于产物的元素浓度, 薄膜的最大厚度受到 加入溶液中反应物的量限制。该方法在应用过程 中溶液的成分控制、 $\mathrm{pH}$ 和温度等因素对薄膜的厚 度、组分和密度等具有重要的影响。近年来该方 法也拓展到了制备金属氧化物。

连续离子层吸附和反应是十九世纪八十年代 发展的一种用来生长金属硫化物和金属氧化物的 方法 ${ }^{33-35}$ 。该方法最早应用于生长单组分的硫化锌 和硫化镉薄膜, 至 90 年代, 科研人员将该方法拓 展应用于生长复合薄膜。该方法是将基底浸没在 含金属阳离子和硫盐的水溶液中。通过逐层的原 子层沉积, 而每一个循环才沉积 $13 \mathrm{~nm}$ 。可见这个 过程非常耗时耗力, 随着科学技术的进展现可以 通过自动化的方式来得到高质量的薄膜。

液相沉积法 36,37 是一种从湿化学法中发展起来 的一种成膜方法, 属于半导体生长工艺的液相外 延生长法, 利用金属氟化物在水中被嗍酸水解作 为前驱体来制备金属氧化物薄膜, 在这个过程中 嗍酸或铝作为金属氟化物的清除剂。与采用化学 浴沉积法制备的金属氧化物相比, 氟化物的清除 剂能更好的控制水解反应的进行和溶液的过饱和
度。这个反应的过程式如下:

$$
\begin{aligned}
& \mathrm{MF}_{n}+m / 2 \mathrm{H}_{2} \mathrm{O} \rightarrow \mathrm{MO}_{m / 2}+n \mathrm{HF} \\
& \mathrm{H}_{3} \mathrm{BO}_{3}+4 \mathrm{HF} \rightarrow \mathrm{BF}_{4}^{-}+2 \mathrm{H}_{2} \mathrm{O}+\mathrm{H}_{3} \mathrm{O}^{+}
\end{aligned}
$$

这一技术主要应用于半导体中金属氧化物的生长 和太阳能电池中。

聚合物辅助沉积法是 2004年由美国 Los Alamos 国家实验室科学家提出的一种薄膜生长方法 ${ }^{38}$ 。经 过十二年的发展已经成功制备出了金属单质 ${ }^{39}$ 、金 属氧化物 ${ }^{40-44}$ 、金属碳化物 ${ }^{45,46}$ 、金属氮化物 ${ }^{47-49}$ 、 金属硫化物等薄膜和复合功能薄膜 ${ }^{50-53}$ 。聚合物辅 助沉积法是一个溶液沉积过程, 它具有化学溶液 法的所有特点。它具有与溶胶凝胶法和其他溶液 法相类似的步骤, 特别之处在于采用水溶性的聚 合物, 该聚合物一般是含胺基的水溶性聚合物如 聚乙烯亚胺、羧化聚乙烯亚胺、磷化聚乙烯亚 胺、磺化聚乙烯亚胺、酰化聚乙烯亚胺、羟基化 水溶性聚乙烯亚胺、聚丙烯酸、聚吡啶、聚乙烯 马来酸等。该类聚合物中的胺基与金属离子直接 络合, 并通过控制热处理的气体氛围能形成高质 量的外延薄膜。

聚合物辅助沉积过程中金属离子与聚合物通 过络合、静电吸引、氢键或共价键的方式形成前 驱体溶液, 前驱体溶液中金属离子能稳定且均匀 的分散在前驱体溶液中。金属离子与聚合物之间 的络合主要有以下几种形式 ${ }^{54}$ : 图 1(a)通过金属离 子的空轨道与聚乙烯亚胺(PEI)中的氮原子上的孤 对电子形成配位键, 从而形成稳定的络合物; 而 对于难络合的金属离子可将聚乙烯亚胺功能化, 如将聚乙烯亚胺上的氨基羧基化、磷酸化等方式 来提高金属与聚合物之间的络合强度, 如图 1(b)所 示; 另外也可以通过先将金属离子与高电荷的螯 合剂反应得到不稳定的阴离子配合物, 再将阴离 子配合物与聚乙烯亚胺反应, 即可得到稳定的络 合物, 如图 1(c)和 1(d)中分别采用乙二胺四乙酸 (EDTA) 和氟化物。以图 1(c) 为例, 金属离子与 EDTA 形成的配合物(M-EDTA)再与聚乙烯亚胺反 应, 这个过程中最大的特点是 EDTA几乎能与所有 的金属离子反应形成配合物, 然后与 PEI 之间通过 静电或氢键的方式形成络合物。

聚合物辅助沉积过程中最主要的特点是金属 离子与聚合物之间的相互作用。其中聚合物具有 以下优点: 抑制了金属离子在溶液中发生不必要 的水解反应; 使得金属离子在溶液中能均匀分 

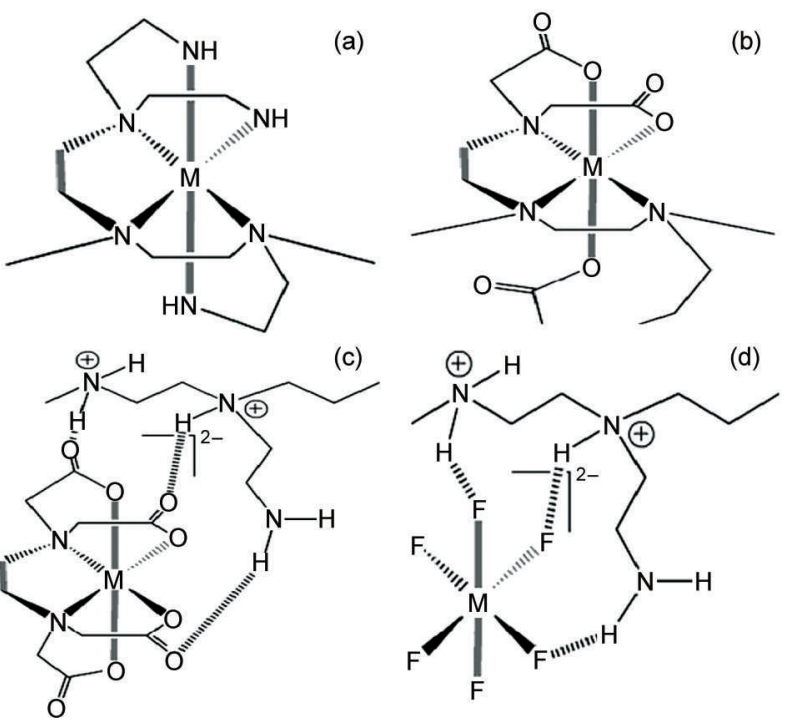

图 1 (a)金属离子与 PEI之间的化学作用示意图; (b)金属 离子与功能化 PEI 之间的化学作用示意图; (c, d) M-EDTA 和 M-F 与 PEI 之间通过氢键和静电作用的示意图 ${ }^{46}$

Fig.1 Schematic illustration of chemical coordination of (a) a metal ion and PEI; (b) metal ion and functionalized PEI; (c, d) EDTA and protonated PEI by hydrogen bonding and electrostatic binding ${ }^{46}$ M: metal ion; PEI: polyethyleneimine; EDTA: ethylene diamine tetraacetic acid

散, 为后期形成均匀的薄膜提供有利条件; 控制 前驱体溶液的粘度能更好地控制薄膜的厚度; 及 维持溶液长期在空气环境下稳定存在。

聚合物辅助沉积过程中超滤是整个过程中不 可或缺的一部分, 对控制溶液的浓度、提纯等方 面起到了重要作用, 因此在聚合物辅助沉积的实 验过程中引入超滤的过程, 装置如图 2 所示。由于 没有络合的阴阳离子的分子量与络合物的分子量 之间存在明显的差异, 通过选择 Amicon filtration 设备中滤膜的分子量就能有效的滤出没有络合上
的阴阳离子。通过超滤的方式就能有效的抑制金 属离子的水解且得到均匀的前驱体溶液。这个过 程对外延薄膜的生长和多元金属化合物的生长就 显得尤为重要。

通过聚合物辅助沉积的方法能制备如图 3 中蓝 色方框内的所有元素的前驱体溶液。对于多元金 属化合物可通过电感偶尔等离子体仪测试各前驱 体溶液中的各元素的浓度, 根据所生长的材料中 元素比例按体积混合即可得到多元的稳定的前驱 体溶液用于成膜。

\section{3 聚合物辅助沉积法制备薄膜的研究进展}

\section{1 聚合物辅助沉积法制备金属氧化物薄膜}

自 2004 年提出聚合物辅助沉积法, 该方法首 先应用于生长金属氧化物薄膜。金属氧化物的生 长先将前驱体溶液通过喷雾、旋涂、喷墨打印、 或提拉涂膜等方式沉积到基底上, 然后将具有预 制膜的基底置于空气中或氧气中高温热处理, 在 热处理的过程中聚合物分解, 聚合物在 $400-$ $500^{\circ} \mathrm{C}$ 基本分解完成, 金属氧化物薄膜开始结晶, 这个结晶的温度会因为材料的不同有一定的差 异, 退火温度也可以控制薄膜生长成单晶、多晶 或非晶结构。到目前为止, 聚合物辅助沉积法已 制备出了二元金属氧化物如氧化锌 $(\mathrm{ZnO}) 、$ 氧化铀 $\left(\mathrm{Eu}_{2} \mathrm{O}_{3}\right)$ 、氧化镍 $(\mathrm{NiO})$ 、氧化钼 $\left(\mathrm{MoO}_{3}\right)^{\text {等 }}{ }^{56-59}$; 三元 金属氧化物如氧化铜铝 $\left(\mathrm{CuAlO}_{2}\right)$ 、钣酸钡 $\left(\mathrm{BaTiO}_{3}\right)$ 、 铁酸钴 $\left(\mathrm{CoFe}_{2} \mathrm{O}_{4}\right)$ 、钛酸锶 $\left(\mathrm{SrTiO}_{3}\right)$ 等 ${ }^{60-63}$; 四元或更 多元金属氧化物如氧化钎钡铜 $\left(\mathrm{YBa}_{2} \mathrm{Cu}_{3} \mathrm{O}_{7-x}\right)$ 、钛酸锶 钡 $\left(\mathrm{Ba}_{1-x} \mathrm{Sr}_{x} \mathrm{TiO}_{3}\right)$ 、氧化锞锶钙锰 $\left(\mathrm{La}_{x} \mathrm{Sr}_{y} \mathrm{Ca}_{1-x-y} \mathrm{MnO}_{3}\right)$ 等 ${ }^{64-67}$ 。

透明导电薄膜如铟掺杂的氧化锡 $\left(\mathrm{In}-\mathrm{SnO}_{2}\right)^{68-70}$ 、

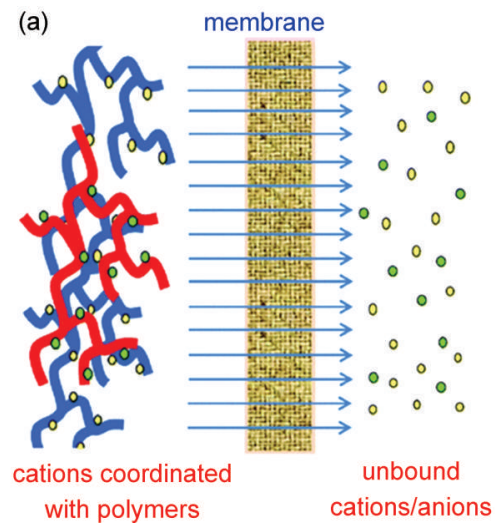

(b)

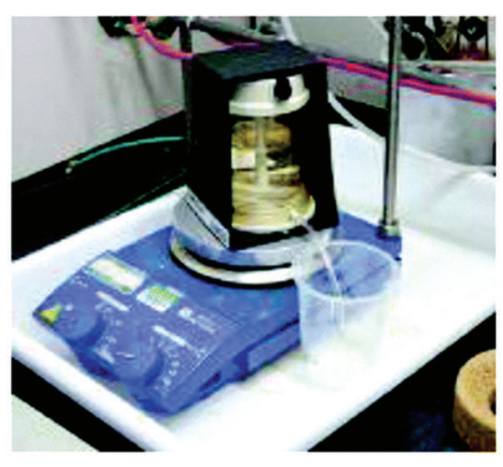

图 2 (a) PAD 过程中的超滤示意图; (b) Amicon Ultrafiltration cell 的实物图 ${ }^{55}$

Fig.2 (a) Schematic illustration of filtration used in the PAD process; (b) photograph of an Amicon Ultrafiltration cell ${ }^{55}$ 


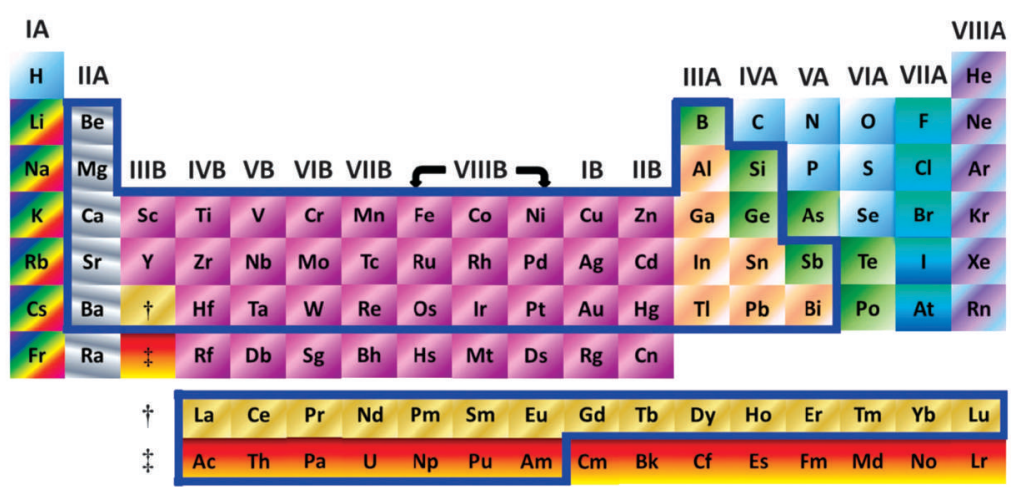

图 3 蓝色方框中的元素能与聚合物形成稳定的前驱体溶液 ${ }^{46}$

Fig.3 Elements in the blue boxes forming a stable precursor with polymer ${ }^{46}$ color online

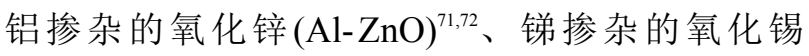
$\left(\mathrm{Sb}-\mathrm{SnO}_{2}\right)^{73-76}$ 等广泛应用在导电电极和玻璃涂层 中, 成为实际应用中非常重要的电极材料。Zou研 究组 ${ }^{59} 2014$ 年采用聚合物辅助沉积法成功的制备 出了透明导电的 $p$ 型氧化镍薄膜(图 4)。氧化镍薄 膜的制备是将氯化镍中的镍离子直接与聚乙烯亚 胺在水中络合形成稳定的络合物, 超滤后旋涂于 氧化铝 $\left(\mathrm{Al}_{2} \mathrm{O}_{3}\right)$ 的单晶基底上, 高温热处理, 在热处
理的过程中在基底的诱导作用下氧化镍外延生长 于 $\mathrm{Al}_{2} \mathrm{O}_{3}$ 基底上, 该方法制备的氧化镍薄膜结晶质 量高, 通过光学测试发现该薄膜在 400-900 nm 的 范围内具有 $>95 \%$ 的高透光性, 薄膜带隙约为 3.71 $\mathrm{eV}$, 通过电学测试得知氧化镍为 $p$ 型半导体。

多元金属氧化物复合薄膜, 如多铁材料, 既 具有电学又有磁学特性, 多功能的复合材料也是 科学和技术领域比较关注的热点材料 ${ }^{77-80}$ 。Luo 研
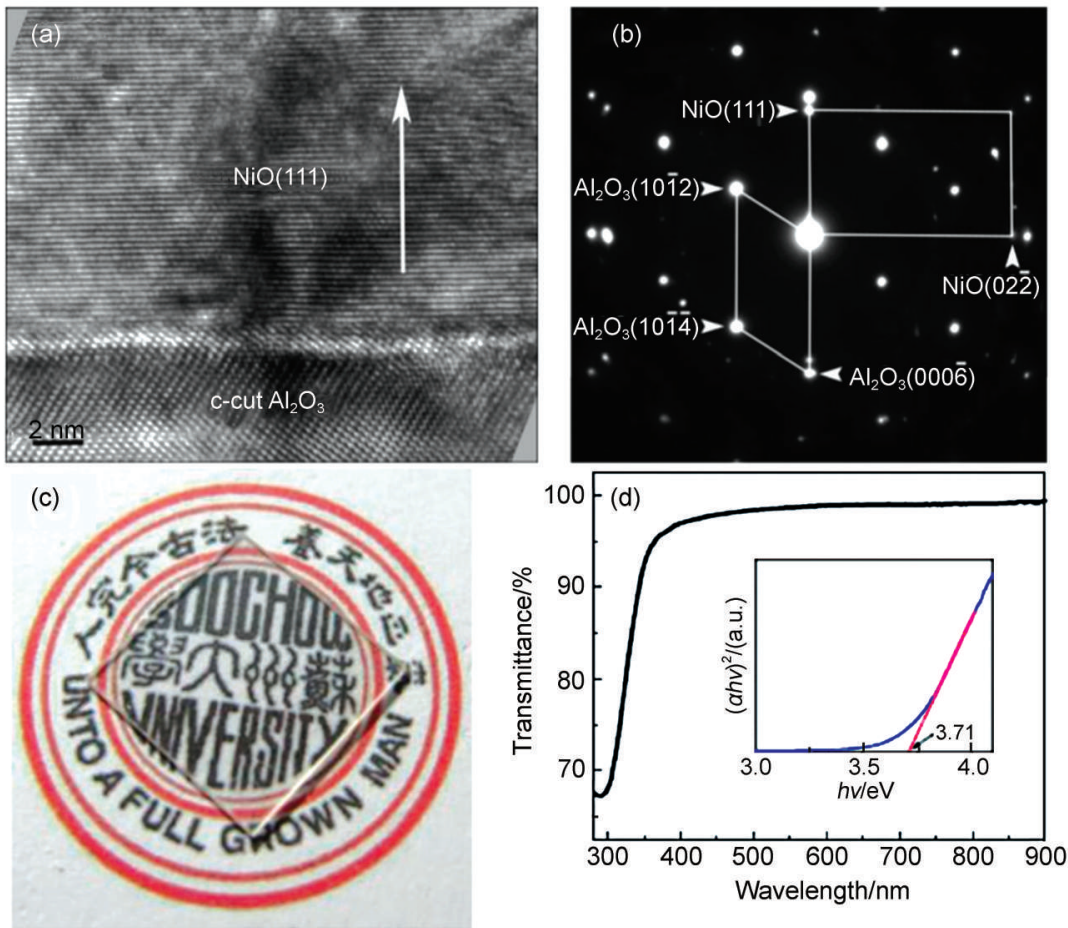

图 4 (a) $\mathrm{NiO}$ 薄膜与 $\mathrm{Al}_{2} \mathrm{O}_{3}$ 基底界面处的高分辨透射电子显微镜(HRTEM)图; (b)与高分辨透射电子显微镜图对应的 $\mathrm{NiO}$ 和 $\mathrm{Al}_{2} \mathrm{O}_{3}$ 选区电子衍射(SAED)图; (c) 薄膜实物放置于带标志的纸上的实物照片; (d) 薄膜在 300-900 $\mathrm{nm}$ 范围内的透射光谱图 ${ }^{59}$ Fig.4 (a) Cross-section high resolution transmission electron microscopy (HRTEM) image taken from the interface between the $\mathrm{NiO}$ thin film and the $\mathrm{Al}_{2} \mathrm{O}_{3}$ substrate; (b) corresponding selected area electron diffraction (SAED) patterns of NiO and

$\mathrm{Al}_{2} \mathrm{O}_{3}$; (c) image of the thin film on a labeled paper; (d) transparency spectrum of the thin film from $300-900 \mathrm{~nm}^{59}$ Inset in (d) shows the plot of $(\alpha h v)^{2}$ versus $h v$. 
究组 ${ }^{81}$ 曾采用聚合物辅助沉积法成功地制备出自组 装的外延 $\mathrm{BaTiO}_{3}-\mathrm{NiFeO}_{3}$ 复合薄膜。通过分别制备 $\mathrm{Ba} 、 \mathrm{Ti} 、 \mathrm{Ni} 、 \mathrm{Fe}$ 四个元素的前驱体溶液, 按照摩 尔比 $1: 1: 1: 1$ 的比例混合, 旋涂成膜高温热处理 后, 成功地将铁电性的 $\mathrm{BaTiO}_{3}$ 嵌入到铁磁性的 $\mathrm{NiFeO}_{3}$ 阵列中(图 5), 使得该复合薄膜既有铁电性 又有铁磁性质(图6)。这有力地说明了聚合物辅助 沉积法中金属离子能均匀的分散在前驱体溶液 中, 且成膜质量高等特点, 这也是其他方法难实 现而聚合物辅助沉积法能简单实现的又一特点。

\section{2 聚合物辅助沉积法制备金属氮化物}

金属氮化物具有高硬度、高熔点、化学性质 稳定等特点, 应用于机械、化工、航天、汽车、 治金等领域; 而其优异的超导性、光电效应等特 点使得在信息技术和能源等领域有着广泛的应 用, 如氮化镓 $(\mathrm{GaN})$ 具有良好的光学性质 ${ }^{82}$, 氮化 钛(TiN) 具有高电导率, 氮化铝(AlN) 具有高电阻率 和良好的透光性 ${ }^{47}$, 氮化铌 $(\mathrm{NbN})$ 具有超导特性 ${ }^{83}$ 。
聚合物辅助沉积法制备金属氮化物的过程与金属 氧化物类似, 将制备金属氧化物的热处理环境从 空气或氧气换成氨气，接下来我们将以两个实例 来说明采用聚合物辅助沉积法能制备出高质量的 金属氮化物薄膜 ${ }^{84,85}$ 。

采用聚合物辅助沉积法在钛酸锶基底上制备 氮化铌薄膜，从图 7 中的高分辨透射电子显微镜图 可知，氮化铌外延生长于基底上。而通过超导性 质的测试发现，生长在钛酸锶基底上的氮化铌薄 膜具有 $14 \mathrm{~K}$ 的超导转变温度, 这些都可以说明采 用聚合物辅助沉积法能得到高质量的外延薄膜。

聚合物辅助沉积法也用于制备多元金属氮化 物，且通过调节前驱体溶液中金属元素的比例可 调控薄膜的组分, 图 8 为 $\mathrm{Al}: \mathrm{Ti}$ 摩尔比为 $1: 1$ 时在 钛酸锶基底上生长的 AlTiN 薄膜即 $\mathrm{Ti}_{0.5} \mathrm{Al}_{0.5} \mathrm{~N}$ 。从 TEM 图可知薄膜厚度约 $90 \mathrm{~nm}$ ，而由于亚晶粒之间 的倾斜使得薄膜中存在不同对比度的亚晶粒。同 时通过精确控制前驱体溶液中 $\mathrm{Al}$ 和 $\mathrm{Ti}$ 的比例能精
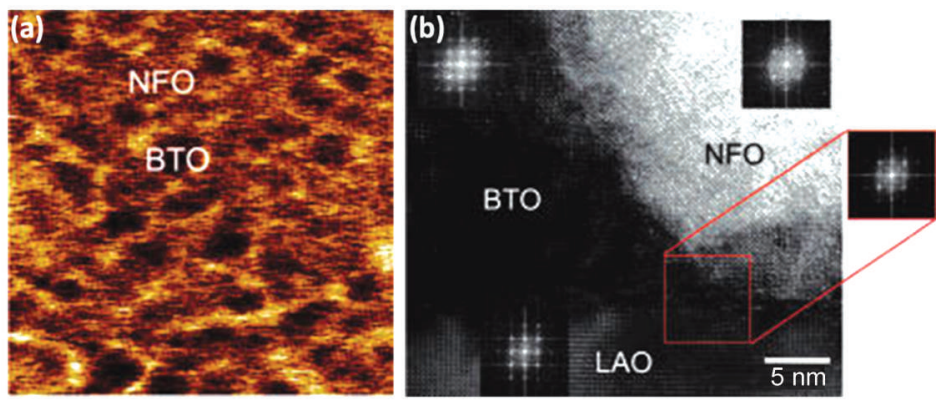

图 5 (a)在 $\mathrm{LaAlO}_{3}$ 基底上生长的 $\mathrm{BaTiO}_{3}-\mathrm{NiFeO}_{3}$ 薄膜的原子力显微镜( $\mathrm{AFM}$ )表面势图 $(1 \mu \mathrm{m} \times 1 \mu \mathrm{m})$;

(b) $\mathrm{BaTiO}_{3}-\mathrm{NiFeO}_{3}$ 薄膜与 $\mathrm{LaAlO}_{3}$ 基底界面处的截面 $\mathrm{HRTEM}^{\text {图 }}{ }^{81}$

Fig.5 (a) Atom force microscopy (AFM) surface potential image of $\mathrm{BaTiO}_{3}-\mathrm{NiFeO}_{3}$ (BTO-NFO) thin film on $\mathrm{LaAlO}_{3}(\mathrm{LAO})$ substrate $(1 \mu \mathrm{m} \times 1 \mu \mathrm{m})$; (b) the cross-section HRTEM image taken from the interface between the $\mathrm{BaTiO}_{3}-\mathrm{NiFeO}_{3}$ thin film and the $\mathrm{LaAlO}_{3}$ substrate ${ }^{81}$
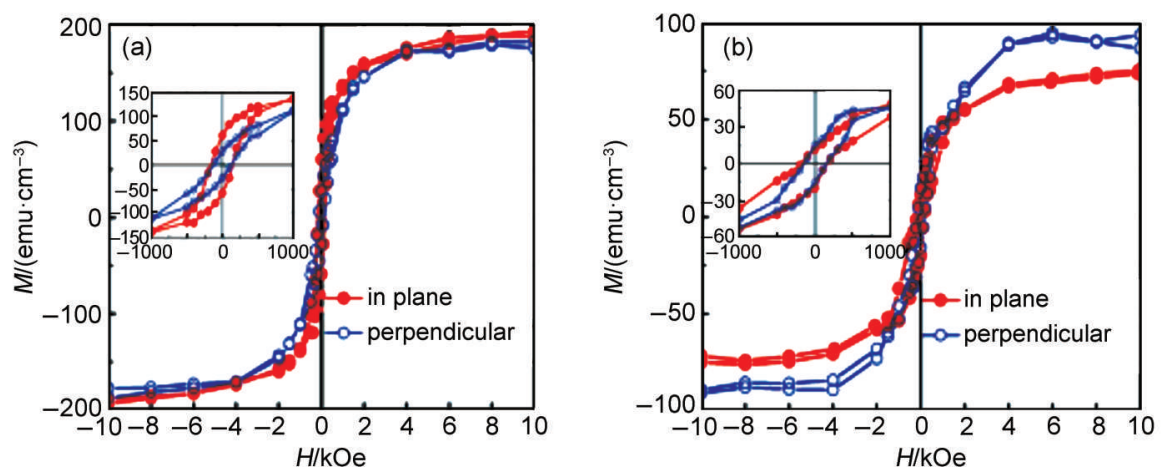

图 6 室温下(a)纯 $\mathrm{NiFeO}_{3}$ 薄膜和(b) $\mathrm{BaTiO}_{3}-\mathrm{NiFeO}_{3}$ 薄膜分别在平行于基底的方向和垂直于基底方向的磁滞回线 ${ }^{81}$

Fig.6 Magnetization versus magnetic field $(M-H)$ hysteresis loops with magnetic field parallel and perpendicular to the substrate at room temperature for (a) pure $\mathrm{NiFeO}_{3}$ thin film and (b) $\mathrm{BaTiO}_{3}-\mathrm{NiFeO}_{3}$ thin film ${ }^{81}$ Insets show the enlarged $M-H$ hysteresis loops. 

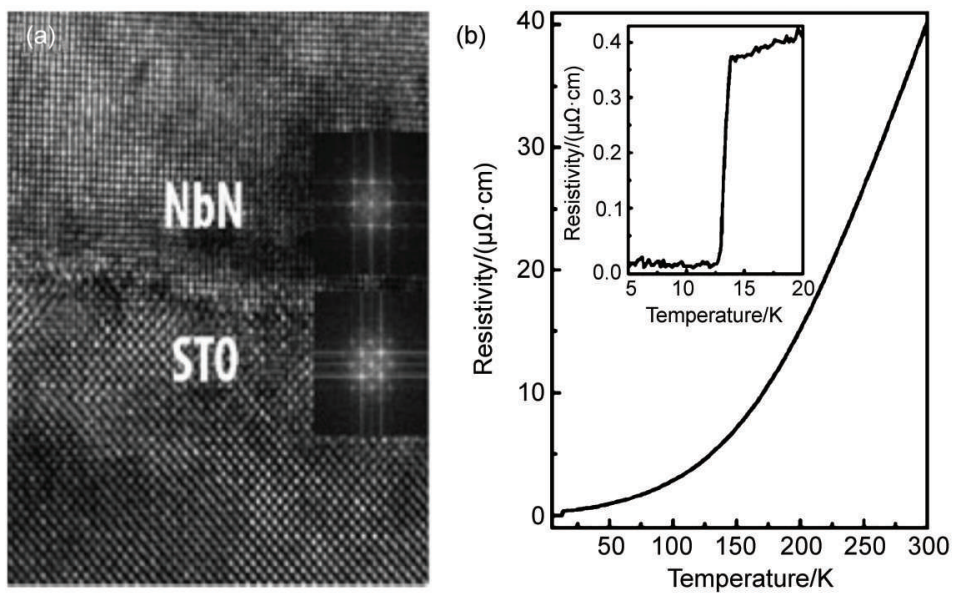

图 7 (a) $\mathrm{NbN}$ 与 $\mathrm{SrTiO}_{3}$ 界面处的截面高分辨透射电子显微镜图; (b)生长在 $\mathrm{SrTiO}_{3}$ 基底上的 $\mathrm{NbN}$ 的超导特性曲线 83

Fig.7 (a) Cross-section HRTEM image taken from the interface between the $\mathrm{NbN}$ thin film and the $\mathrm{SrTiO}_{3}$ (STO) substrate;

(b) superconducting properties of $\mathrm{NbN}$ grown on $\mathrm{SrTiO}_{3}$ substrate ${ }^{83}$

Insets in (a) present FFTs of $\mathrm{NbN}$ and STO. Inset in (b) displays an enlarged temperature dependent resistivity from 5 to $20 \mathrm{~K}$.

确调控薄膜成分，如图9所示，通过研究发现不同 $\mathrm{Al}$ 和 $\mathrm{Ti}$ 的比例能调控薄膜的透光性, 随着 AlTiN 薄膜中 $\mathrm{Al}$ 含量的增加透光性提高, 同样地, $\mathrm{Al}$ 和 $\mathrm{Ti}$ 的比例对 AlTiN 薄膜的电阻率也有一定程度的调 控。从图中可知纯 TiN 具有良好的导电性, 其电阻 率在室温时为 $1.9 \times 10^{-5} \Omega \cdot \mathrm{cm}$, 而通过电导率与温 度的变化曲线可知纯 $\mathrm{TiN}$ 具有金属特性, 而通过加 入一定量的 $\mathrm{Al}$ 薄膜显示半导体特性。从这些结果
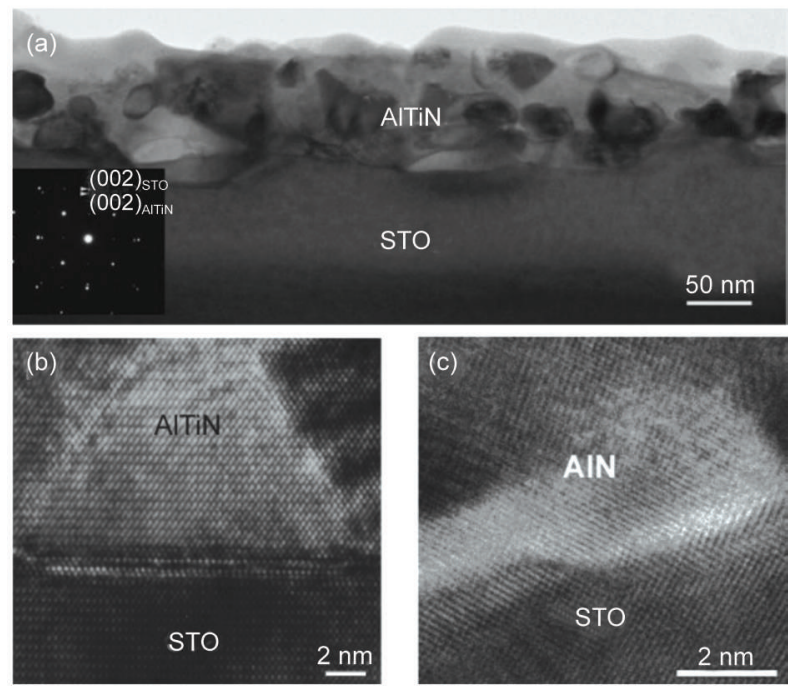

图 8 (a)生长在钛酸锶基底上的 $\mathrm{Ti}_{0.5} \mathrm{Al}_{0.5} \mathrm{~N}$ 的 TEM图;

(b) $\mathrm{Ti}_{0.5} \mathrm{Al}_{0.5} \mathrm{~N}$ 和 $\mathrm{SrTiO}_{3}$ 界面处的截面 HRTEM 图;

(c) AIN 和 $\mathrm{SrTiO}_{3}$ 界面处的截面 HRTEM 图 ${ }^{47}$

Fig.8 (a) TEM image of a $\mathrm{Ti}_{0.5} \mathrm{Al}_{0.5} \mathrm{~N}$ (AITiN) film on $\mathrm{SrTiO}_{3}$ (STO), (b) the cross-section HRTEM image from the interface of $\mathrm{Ti}_{0.5} \mathrm{Al}_{0.5} \mathrm{~N}$ and $\mathrm{SrTiO}_{3}$, and (c) cross-section HRTEM image from the interface of $\mathrm{AIN}$ and $\mathrm{SrTiO}_{3}{ }^{47}$
可知通过金属元素比例的调节可以调控薄膜的透 光性和电学特性。

\section{3 聚合物辅助沉积法制备金属碳化物}

金属碳化物多具有金属光泽、导电性好、硬 度大等特点，常被用作金属合金的原料，另外金 属碳化物还具有其他的特性如高温稳定、耐腐 蚀、抗氧化等特点。这些特点使得金属碳化物在 可穿戴涂层、钝化层、高温电子器件等方面具有 非常吸引人的应用前景。因此生长具有高硬度、 高电导率、化学稳定、高质量的金属碳化物外延 薄膜显得尤为重要 ${ }^{86-88}$, 需发展一种通用的方法来 制备金属碳化物使得金属碳化物能在科学技术领 域得到广泛的应用。在此, 聚合物辅助沉积法成 功地制备了各种金属碳化物薄膜。金属碳化物的 薄膜生长过程与生长金属氮化物的方法类似, 仅 是将热处理环境从氨气气氛换成乙烯气氛。

碳化硅是一种宽带隙半导体, 其具有化学性 质稳定、导热系数高、热膨胀系数小、耐磨性能 好, 且硬度仅次于金刚石等特点 ${ }^{89,90}$ 。由于其耐高 温且宽带隙等优点使得碳化硅能应用于高温太阳 能电池中作为窗口层, 应用于航天航空等领域。 采用聚合物辅助沉积法成功地在单晶硅上生长出 外延碳化硅薄膜。有两种方法制备硅的前驱体溶 液, 一种是利用硅酸盐自身直接与聚合物(聚乙烯 亚胺)之间通过氢键和静电等方式络合, 另外一种 是采用硅离子与氟离子配位, 再与聚乙烯亚胺络 合, 这一过程与聚合物辅助沉积法中的图 1(d)相对 应。通过这两种制备前驱体溶液的方法均能得到 

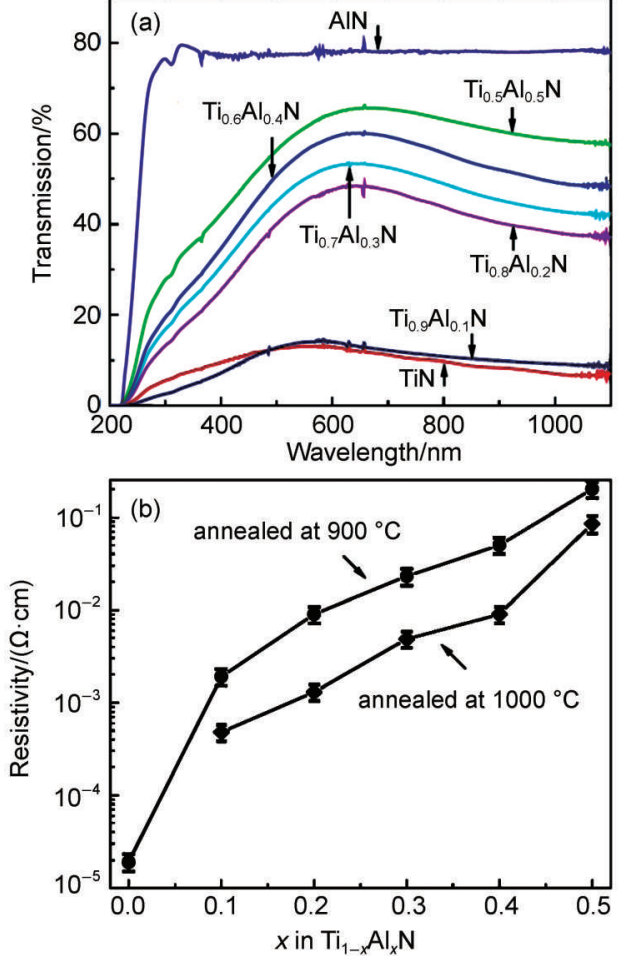

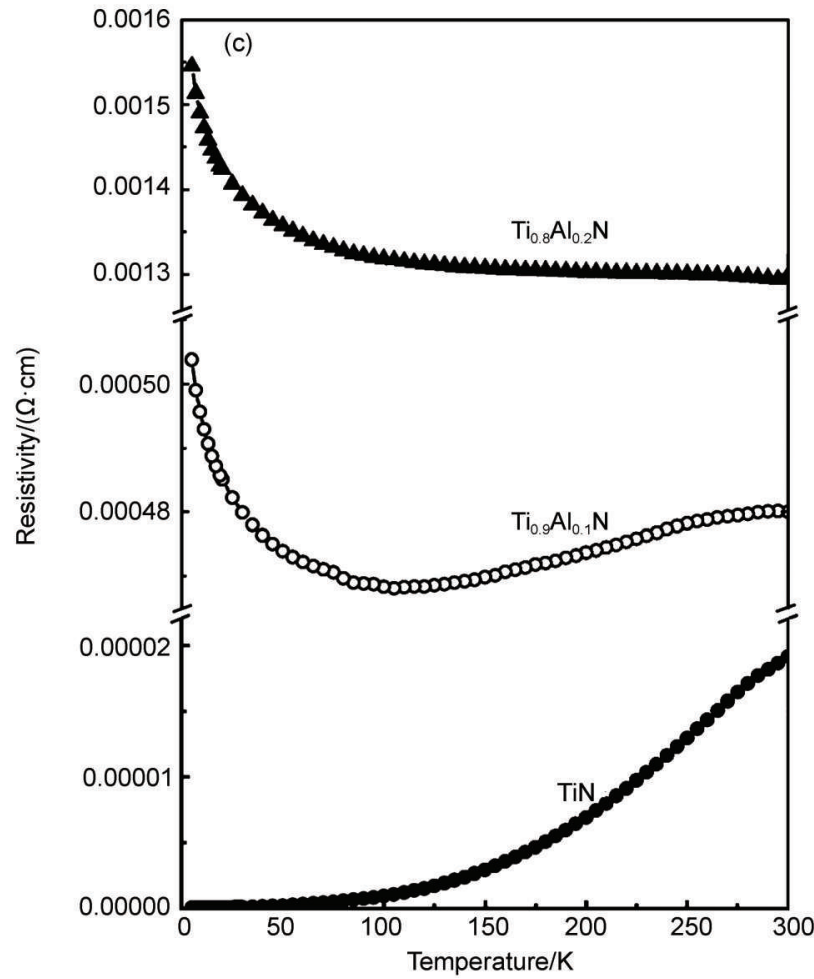

图 9 (a) $\mathrm{Ti}_{1-x} \mathrm{Al} \mathrm{I}_{x} \mathrm{~N}(x=0-0.5,1)$ 薄膜的透光性曲线; (b) 在 900 和 $1000{ }^{\circ} \mathrm{C}$ 退火后的 $\mathrm{Ti}_{1-x} \mathrm{Al}_{x} \mathrm{~N}(x=0-0.5)$ 薄膜在室温下的 电阻率; (c)于 $1000{ }^{\circ} \mathrm{C}$ 退火的 $\mathrm{SrTiO}_{3}$ 基底上生长的 $\mathrm{Ti}_{1-x} \mathrm{Al}_{x} \mathrm{~N}(x=0,0.1,0.2)$ 薄膜随温度变化的电阻率曲线 ${ }^{47}$

Fig.9 (a) Optical transmittance spectra of $\mathrm{Ti}_{1-x} \mathrm{Al}_{x} \mathrm{~N}(x=0-0.5$, 1) thin film; (b) room temperature resistivity of $\mathrm{Ti}_{1-x} \mathrm{Al}_{x} \mathrm{~N}(x=0-0.5)$ thin film annealed at 900 and $1000{ }^{\circ} \mathrm{C} ;(\mathrm{c})$ temperature-dependent resistivity of a $\mathrm{Ti}_{1-x} \mathrm{Al}_{x} \mathrm{~N}(x=0,0.1,0.2)$ film on $\mathrm{SrTiO}_{3}$ substrate annealed at $1000{ }^{\circ} \mathrm{C}^{47}$

稳定的前驱体溶液。图 10 所示为生长在硅(111)基 底上的碳化硅薄膜的 X射线衍射花样图和高分辨 透射电子显微镜图，从图中可得知碳化硅外延生 长于硅基底上, 这也是采用化学溶液法生长碳化 硅的一次突破。

碳化钛薄膜具有硬度大，导电性好等特点， 但是生长碳化钛薄膜的方式仅限于物理气相沉积 和化学气相沉积过程, 因此提出一种设备简单成 本低廉, 成膜面积大的方式就非常具有优势, 在 此聚合物辅助沉积法能成功的制备出高质量的碳 化钛薄膜 ${ }^{91}$ 。从图 11 可知, 碳化钛薄膜外延生长于 氧化铝基底上, 通过测试薄膜的硬度和杨氏模量 得知其硬度为 $21.27 \mathrm{GPa}$, 杨氏模量为 $413 \mathrm{GPa}$ 。通 过测试其电阻率随温度的变化曲线发现碳化钛薄 膜呈半导体特性, 总之, 通过实验发现采用聚合 物辅助沉积法制备出了高质量的碳化钛外延薄膜。

\section{4 聚合物辅助沉积法制备金属单质}

由于锗的化合物要么不稳定要么很难溶于 水, 因此制备单质锗薄膜就显得很困难。聚合物 辅助沉积法于 2009 年首次成功地在单晶硅基底上
制备出了外延锗薄膜。生长的锗薄膜具有高结晶 性且薄膜表面非常平整。通过物性测试发现锗薄 膜在室温下具有高的迁移率 $\left(1700 \mathrm{~cm}^{2} \cdot \mathrm{V}^{-1} \cdot \mathrm{s}^{-1}\right)$ 和高 的载流子浓度 $\left(3.45 \times 10^{19} \mathrm{~cm}^{-3}\right)$ (图 12)。可见通过聚 合物辅助沉积法为金属单质薄膜的制备提供了一 种可行的方法。

\section{5 聚合物辅助沉积法制备金属硫化物/硒化物}

近年来, 金属硫/硒化物在燃料电池、发光二 极管、催化、传感器、锂离子电池、太阳能电 池、超级电容器、热电器件和存储器件中得到了 广泛的应用 ${ }^{92-97}$ 。各种制备金属硫化物/硒化物的方 法也不断的被提出, 但是大部分的方法会采用一 些有害的有机溶剂或者制作过程复杂等限制, 使 得这些方法没有得到推广。因此提出一些制备过 程简单环保的方法来制备金属硫/硒化物就显得尤 为重要, 而聚合物辅助沉积法是一种采用水为溶 剂的绿色环保的方法, 通过该方法我们成功的制 备出了硫化钼、硫化锌、硫化铼、铜锌锡锗硫硒 等金属硫化物和金属硒化物。

铜锌锡硫硒是一种具有窄带隙 $(1.0-1.5 \mathrm{eV})$ 、 

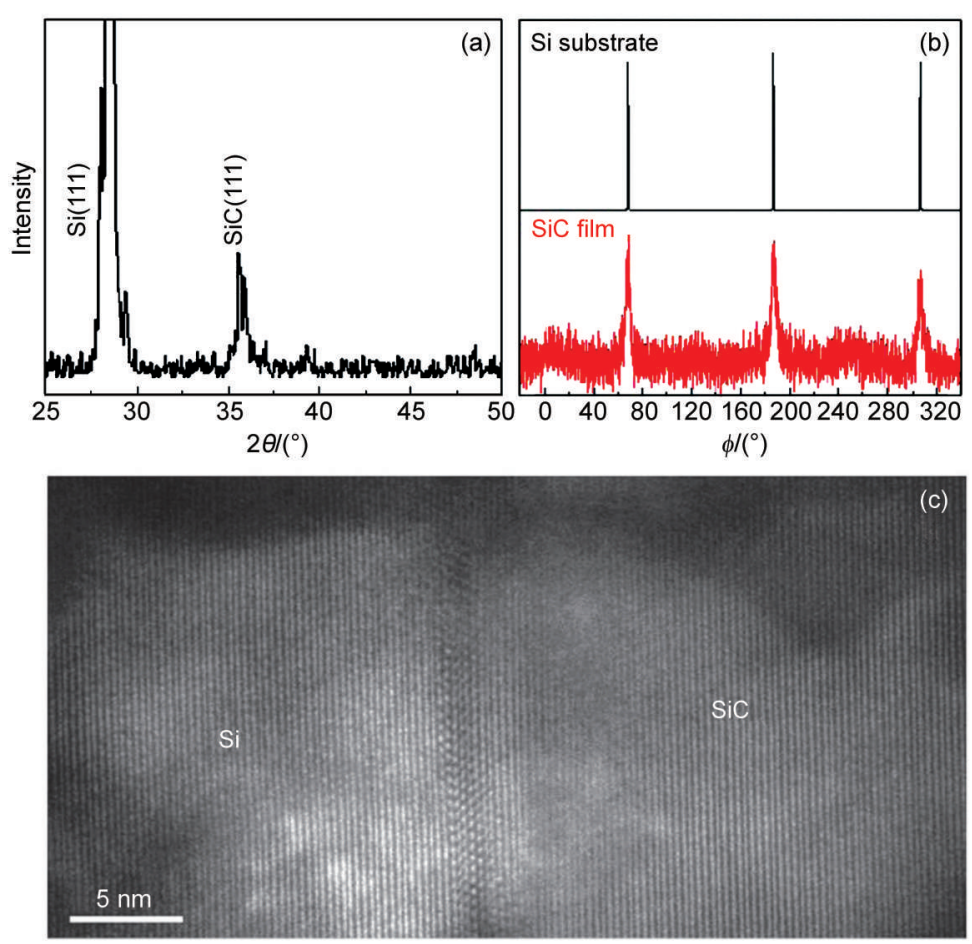

图 $10(a, b)$ 生长在硅(111)基底上的碳化硅薄膜的 X 射线衍射(XRD)图;

(c)生长在硅(111)基底上的碳化硅薄膜的高分辨透射电子显微镜图 ${ }^{46}$

Fig.10 (a, b) X-ray diffraction (XRD) patterns of SiC thin film on Si(111) substrate;

(c) HRTEM image of the $\mathrm{SiC}$ thin film on $\mathrm{Si}(111)$ substrate $^{46}$
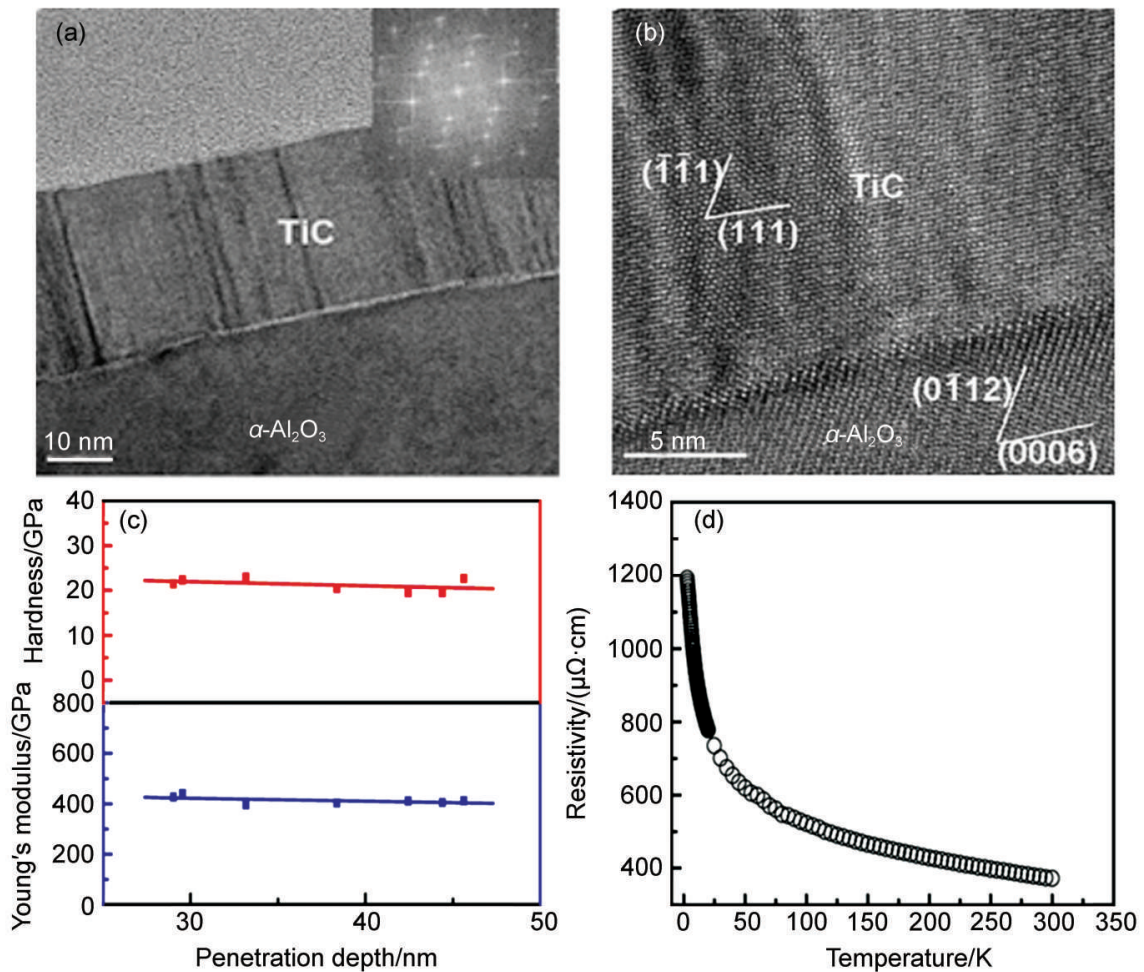

图 11 (a) $\mathrm{TiC}$ 薄膜与 $\mathrm{Al}_{2} \mathrm{O}_{3}$ 基底界面处截面的 TEM 图; (b) $\mathrm{TiC}$ 薄膜与 $\mathrm{Al}_{2} \mathrm{O}_{3}$ 基底界面处截面的 HRTEM 图,

(c)硬度与杨氏模量随渗透深度的曲线图; (d)电阻率随温度变化的曲线图 ${ }^{91}$

Fig.11 (a) Cross-section TEM image and (b) cross-section HRTEM image of the interface between TiC thin film and $\mathrm{Al}_{2} \mathrm{O}_{3}$ substrate; (c) the hardness and Young's modulus as a function of penetration depth;

(d) the resistivity as a function of temperature ${ }^{91}$ 
(a)
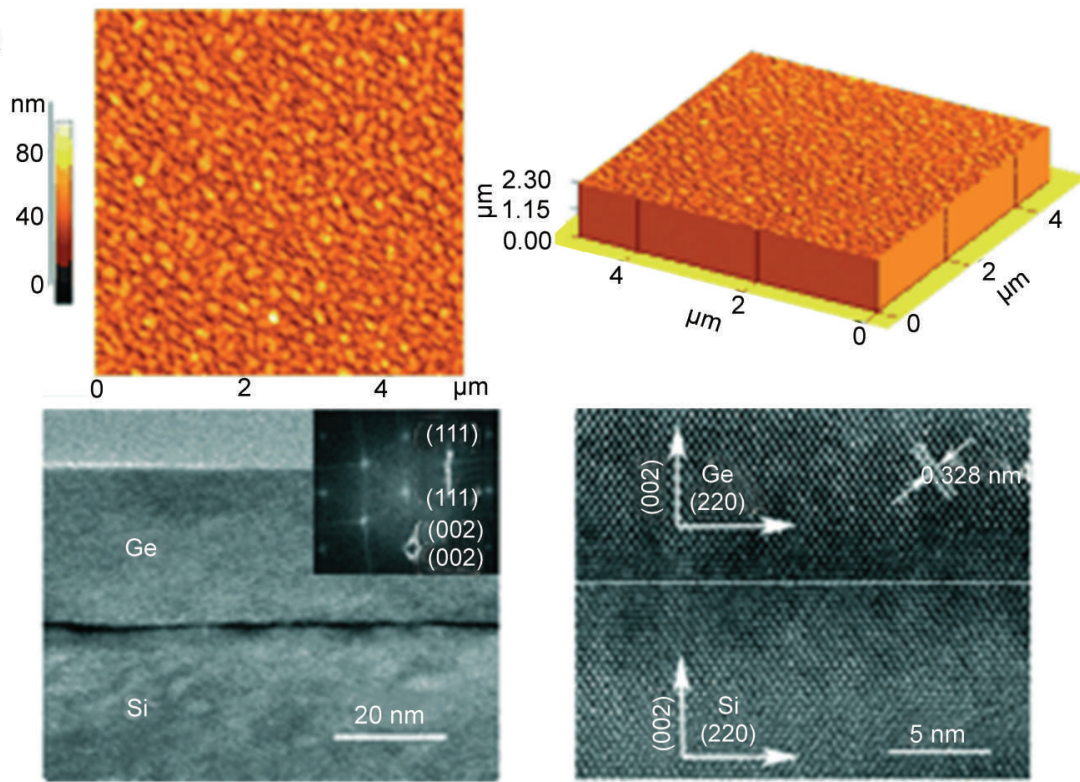

(b)

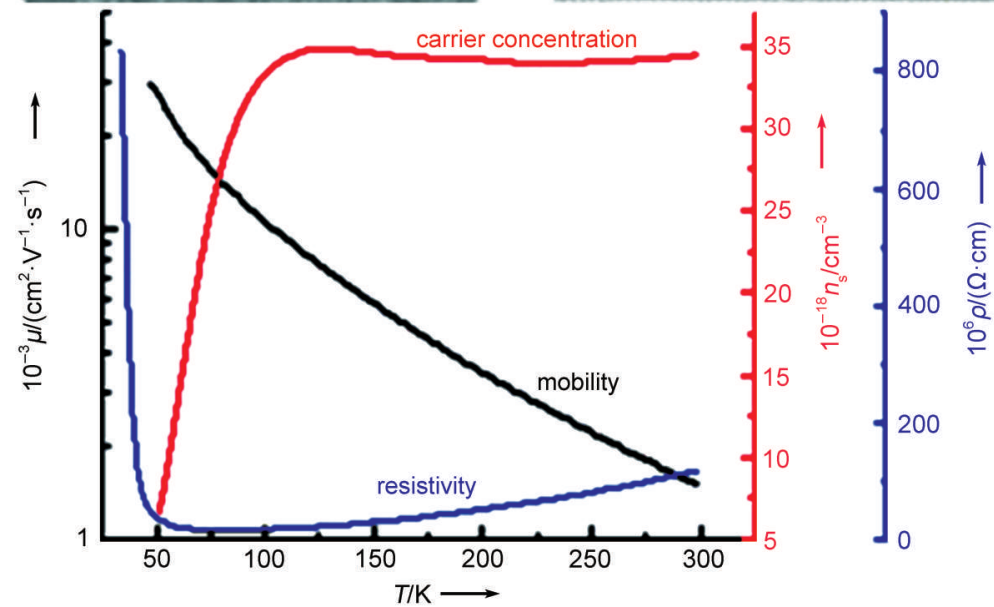

图 12 (a)薄膜的表面和界面微结构图; (b) 外延锗薄膜的物理性质 ${ }^{39}$

Fig.12 (a) Surface and interface microstructures of films; (b) transport properties of epitaxial Ge films ${ }^{39}$

高吸光系数、无毒、元素含量丰富的 $p$ 型半导体， 被应用于太阳能电池领域中取代硫化镉等有毒材 料 ${ }^{98-100}$ 。通过聚合物辅助沉积法采用固体硫源和硒 源在氩气气氛下热处理即可得到铜锌锡硫 (CZTS)、铜锌锡硒(CZTSe)、铜锌锡硫硒(CZTSSe) 等化合物薄膜。图 13 所示为得到的产物的 XRD 图 和采用聚合物辅助沉积法制备的铜锌锡硫硒用于 太阳能电池中作为吸收层的太阳能电池的电流密 度一电压曲线。

\section{6 聚合物辅助沉积法制备复合功能薄膜}

碳材料如石墨烯、碳纳米管等具有优异的电 学性能被广泛的应用于薄膜材料中形成复合功能 薄膜, 提高薄膜的性质 ${ }^{101-103}$ 。碳化铌 $(\mathrm{NbC})$ 与碳纳 米管的复合功能薄膜(图 14) $)^{104}$, 通过将碳纳米管引
入至薄膜中, 能提高薄膜的上转变场。同时不仅 可以提高薄膜的超导转变温度, 还可以提高薄膜 的拉伸强度。

\section{7 采用聚合物辅助沉积法在不规则基底上制备 薄膜}

经过长期的聚合物辅助沉积法的应用发现聚 合物辅助沉积法生长薄膜的方式是一种自下而上 的生长方式。金属络合物的前驱体溶液旋涂在基 底上, 前驱体溶液吸附在基底表面, 经过热处理 的最后一步才会使得金属络合物断开, 这样一个 过程使得该方法能应用于生长多孔/复杂 3-D 结构 的不规则的涂层, 这也是很多物理方法和化学气 相沉积很难实现的一种结构。图 15 为有/无 $\mathrm{ZrO}_{2}$ 涂 层的 Anodiscs ${ }^{\mathrm{TM}}$ 的 SEM 图, 而通过气体和液体通 

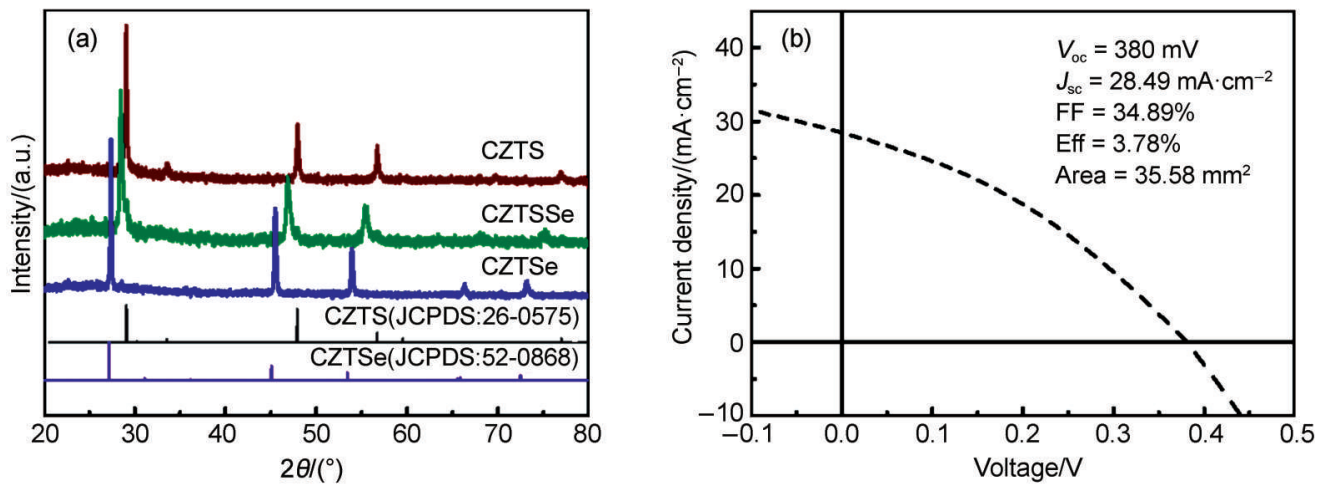

图 13 (a) CZTS, CZTSSe, CZTSe 薄膜的 XRD 图;

(b)基于 CZTSSe 作为活性层的太阳能电池的电流密度一电压 $(J-V)$ 曲线图

Fig.13 (a) XRD patterns of CZTS, CZTSSe, CZTSe thin films;

(b) current density-voltage $(J-V)$ curve for the device based on a CZTSSe active layer
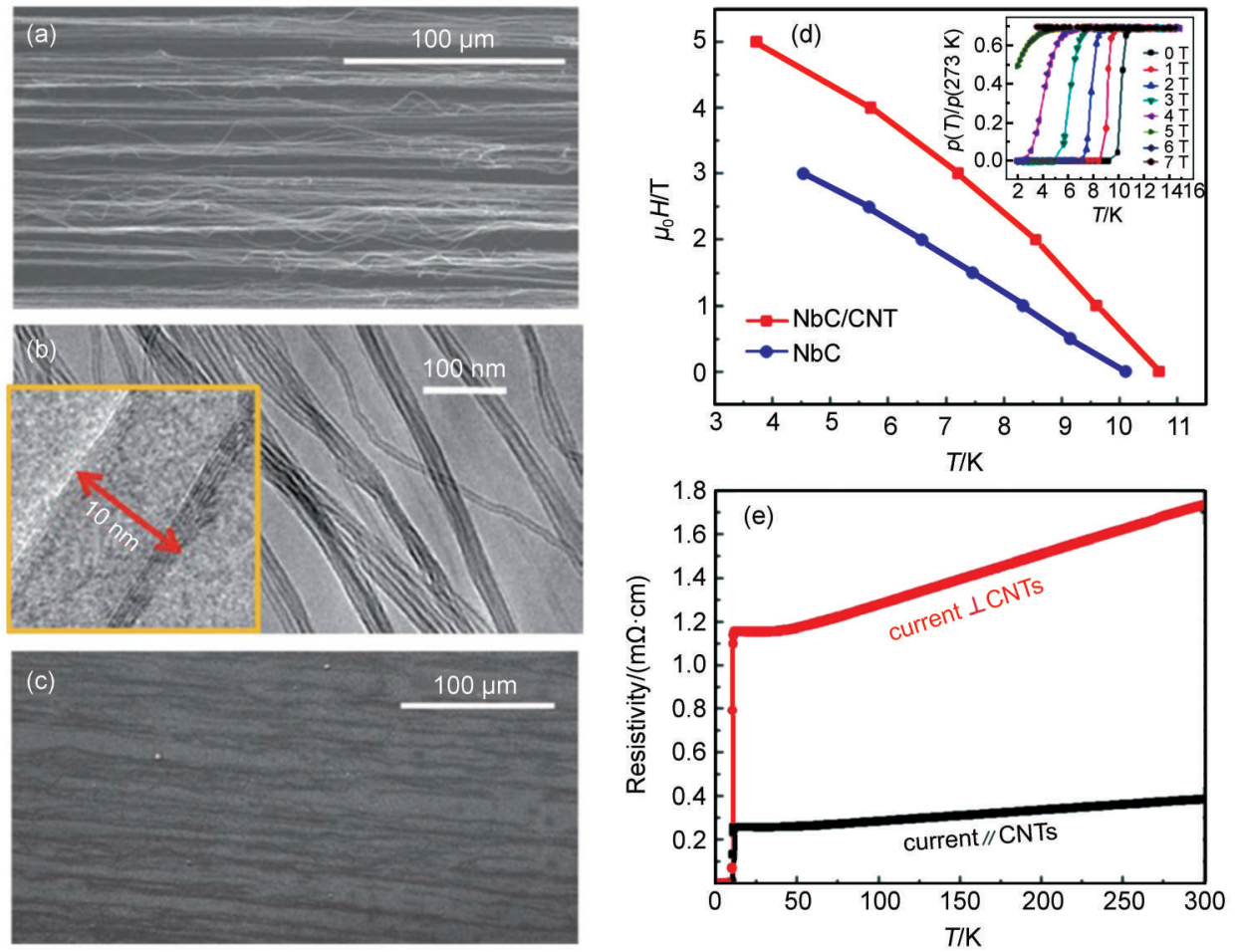

图 14 (a) CNT 的扫描电子显微镜(SEM)图; (b) CNT 的 TEM 图; (c) NbC/CNT 复合薄膜的 SEM 图; (d) $\mathrm{NbC/CNT}$ 复合薄膜的上临界场随温度的变化曲线; (e) NbC/CNT 复合薄膜的电阻率随温度的变化曲线 ${ }^{104}$

Fig.14 (a) Scanning electron microscope (SEM) image of CNT, (b) TEM image of CNT; (c) SEM image of $\mathrm{NbC/CNT}$ composite thin film; (d) temperature vs upper critical field of NbC/CNT composite thin film; (e) temperature dependence of the resistivities of $\mathrm{NbC} / \mathrm{CNT}$ composite thin film ${ }^{104}$

过薄膜的流动测试发现, 有无 $\mathrm{ZrO}_{2}$ 涂层对滤膜的 性能无明显的影响, 但是通过化学稳定性测试发 现, 涂有 $\mathrm{ZrO}_{2}$ 涂层的滤膜在 $\mathrm{pH}$ 为 12.4 的溶液中能 存放 $24 \mathrm{~h}$ 而无 $\mathrm{ZrO}_{2}$ 涂层得滤膜浸泡 $15 \mathrm{~min}$ 就会被 破坏, 可见通过在滤膜上加入 $\mathrm{ZrO}_{2}$ 涂层能明显的 提高滤膜的化学稳定性 ${ }^{25}$ 。

\section{4 未来聚合物辅助沉积法的挑战}

对于半导体薄膜而言，薄膜中的杂质对薄膜 的电学性质具有明显的影响, 而我们的实验发现 在有机物分解过程中会残留一定量的碳, 如生长 在 $\mathrm{LaAlO}_{3}$ 基底上的 $\mathrm{SrTiN}_{2}$ 薄膜的卢瑟福背散射图 谱(图 16)可知, $\mathrm{SrTiN}_{2}$ 薄膜中含有微量的碳。尽管 部分薄膜对碳的含量要求非常高, 但是有部分薄 


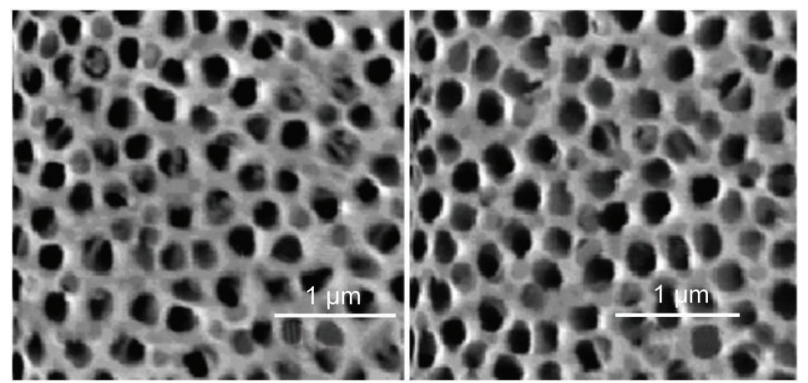

图 15 无涂层(左)和有 $\mathrm{ZrO}_{2}$ 涂层(右)的 $\operatorname{anodisc}^{\mathrm{TM}}$ 扫描电子显微镜图 ${ }^{25}$

Fig.15 SEM iamges of uncoated (left) and $\mathrm{ZrO}_{2}$ coated (right) anodisc ${ }^{\mathrm{TM} 25}$

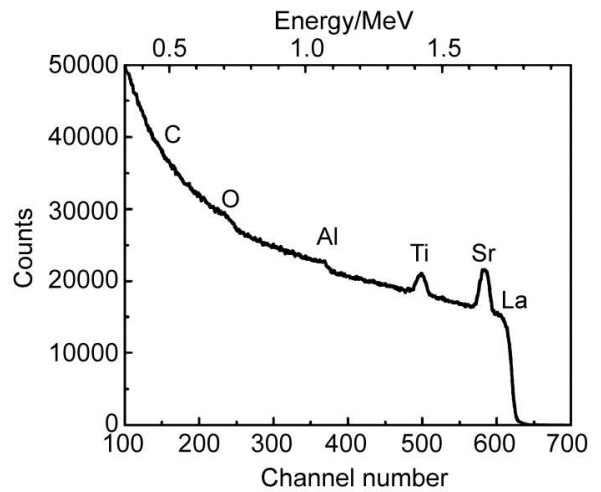

图 16 生长在 $\mathrm{LaAlO}_{3}$ 基底上的 $\mathrm{SrTiN}_{2}$ 薄膜的 卢瑟福背散射图谱 ${ }^{46}$

Fig.16 Rutherford back scattering (RBS) spectrum of a $\mathrm{SrTiN}_{2}$ film on $\mathrm{LaAlO}_{3}$ substrate $^{46}$

膜却希望含有一定量的碳从而能提高薄膜的性 能, 如含有一定量的碳会提高 $\mathrm{MgB}_{2}$ 的超导温度 ${ }^{105}$ 。

另一挑战是与热处理相关的一个问题, 在聚合 物辅助沉积法生长薄膜的过程中, 由于聚合物分解 的温度在 $400-500{ }^{\circ} \mathrm{C}$, 且薄膜的结晶温度甚至比 聚合物的裂解温度更高这就使得薄膜的生长热处理 的温度会相对较高, 也就是说基底的耐热温度要高 于薄膜的生长温度, 这就会限制基底的选择, 对于 该方法的应用领域也会受到一定的限制。

\section{5 结论和展望}

聚合物辅助沉积法是溶液沉积法中的一种, 可直接将化学溶液应用于某表面即可。聚合物辅 助沉积法区别于其他方法的地方在于整个过程中 采用一种特殊的聚合物, 该聚合物可以阻止金属 离子发生不必要的化学反应, 保持溶液稳定, 确 保薄膜均匀且具有优良的物理性能。换言之, 每 个稳定的金属离子会与一个阴离子反应形成一个
络合物。另外, 聚合物辅助沉积过程能简单地通 过需要生长材料的化学组分来混合不同的金属前 驱体溶液得到最终的前驱体溶液, 即可精确地控 制最终溶液和薄膜的组分。

聚合物辅助沉积法不仅可以生长单相的金属氧 化物、金属碳化物、金属氮化物、金属单质、金属 硫/硒化物薄膜, 也可以生长如 $\mathrm{NiFeO}_{3} / \mathrm{BaTiO}_{3}$ 、 $\mathrm{SiO}_{2} / \mathrm{CoFe}_{2} \mathrm{O}_{4} 、 \mathrm{CNT} / \mathrm{NbC}$ 等复合功能薄膜, 还可以 在不规则基底上生长薄膜甚至任何尺寸和形状的 平面或 3-D 结构。即聚合物辅助沉积法可以形成完 美结构的薄膜, 纳米粒子、管状物的内部表面等 结构。总之, 聚合物辅助沉积法在生长薄膜材料 方面取得了丰硕的成果，有望进一步应用于先进 半导体和光电器件领域。

\section{References}

(1) Wang, H.; Xu, H. Y.; Yan, H. Funct. Mater. Information 2006, 3, 1. [汪 浩, 徐海燕, 严 辉. 功能材料信息, 2006, 3, 1.]

(2) Janotti, A.; Walle, C. Rep. Prog. Phys. 2009, 72, 126501. doi: 10.1016/S0040-6090(00)01369-9

(3) Willander, M.; Friesel, M.; Wahab, Q.; Staumal, B. J. Mater: Sci.: Mater. Electron. 2006, 17, 1. doi: 10.1007/s10854-0055137-4

(4) Pawar, S. M.; Pawar, B. J.; Kim, H.; Joo, S.; Lokhande, C. Curr. Appl. Phys. 2011, 11, 117. doi: 10.1016/j.cap.2010.07.007

(5) Shou, C. H.; Luo, Z. Y.; Wang, T.; Shen, W. D.; Rosengarten, G.; Wei, W.; Wang, W.; Ni, M. J.; Cen, K. F. Appl. Energy 2012, 92, 298. doi: 10.1016/j.apenergy.2011.09.028

(6) Tougas, I. M.; Gregory, O. J. Sensors 2013, 13, 15324. doi: $10.3390 / \mathrm{s} 131115324$

(7) Nechache, R.; Huang, W.; Li, S.; Rosei, F. Nanoscale 2016, 8, 3237. doi: 10.1039/C5NR08819D

(8) Cao, D. W.; Nasori, N.; Wang, Z. J.; Mi, Y.; Wen, L.Y.; Yang, Y.; Qu, S. C.; Wang, Z. G.; Lei, Y. J. Mater. Chem. A 2016, 4, 8995. doi: 10.1039/C6TA01234E

(9) Park, H.; Kim, H.; Moon, G.; Choi, W. Y. Energy Environ. Sci. 2016, 9, 411. doi: 10.1039/C5EE02575C

(10) Scragg, J.; Waitjen, J. T.; Edoff, M.; Ericson, T.; Kubart, T.; Björkman, C. P. J. Am. Chem. Soc. 2012, 134, 19330. doi: $10.1021 / \mathrm{ja} 308862 \mathrm{n}$

(11) Attia, M. S.; Zoulghena, M.; Abdelmottaleb, S. Analyst 2013, 139, 793. doi: 10.1039/C3AN01645E

(12) Voorst, P. D.; Wit, M. R.; Offerhaus, H. L.; Tay, S.; Thomas, J.; Peyghambarian, N.; Boller, K. J. Opt. Express 2007, 15, 17587. doi: $10.1364 /$ OE. 15.017587

(13) Li, X. S.; Zhu, Y. W.; Cai, W. W.; Borysiak, M.; Han, B. Y.; Chen, D.; Piner, R. D.; Colombo, L.; Ruoff, R. S. Nano Lett. 2009, 9, 4359. doi: 10.1021/n1902623y 
(14) Meenakshi, P.; Karthick, R.; Selvaraj, M.; Ramu, S. Sol. Energy Mater. Sol. Cells 2014, 128, 264. doi: 10.1016/j. solmat.2014.05.013

(15) Loh, K. P.; Bao, Q.; Eda, G.; Chhowalla, M. Nat. Chem. 2010, 2, 1015. doi: 10.1038/nchem.907

(16) Jin, S.; Tiefel, T. H.; McCormack, M.; Fastnachtm, R. A.; Ramesh, R.; Chen, L. H. Science 1994, 264, 413. doi: 10.1126/ science264.5157.413

(17) Yourdkhani, A.; Caruntu, G. J. Phys. Chem. C 2011, 115, 14797. doi: 10.1021/jp202127t

(18) Xie, W. W.; Luo, H. X.; Baroudi, K.; Krizan, J. W.; Phelan, B. F.; Cava, R. J. Chem. Mater. 2015, 27, 1149. doi: 10.1021/ cm504449s

(19) Lin, S. Y.; Chen, Y. C.; Wang, C. M.; Hsieh, P. T.; Shih, S. C. Appl. Surf. Sci. 2009, 255, 3868. doi: 10.1016/j. apsusc.2008.10.069

(20) Pires, A. L.; Belo, I. T.; Gomes, L.; Fernandes, P. B.; Tavares, A. M. L.; Lopes, A. M.; Pereira, J. P. Mater. Today: Proc. 2015, 2, 26. doi: 10.1016/j. matpr.2015.04.004

(21) Hu, M.; Zeng, Q.; Zhu, M. Electrochim. Acta 2009, 54, 2843. doi: 10.1002/pssa.200881796

(22) Elangovan, E.; Martins, R.; Fortunato, E. Phys. Status Solidi A 2009, 206, 2123. doi: 10.1002/pssa.200881796

(23) Bhosle, V.; Tiwari, A.; Narayan, J. J. Appl. Phys. 2005, 97, 083539. doi: $10.1063 / 1.1868852$

(24) Hass, D.; Marciano, Y.; Wadley, H. Surf. Coat. Technol. 2004, 185, 283. doi: 10.1016/j.surfcoat.2003.12.027

(25) Burrell, A.; McCleskey, T.; Jia, Q. X. Chem. Commun. 2008, 1271. doi: 10.1039/B712910F

(26) Barber, Z. H. J. Mater. Chem. 2006, 16, 334. doi: 10.1039/ B506228D

(27) Hench, L. L.; West, J. K. Chem. Rev. 1990, 90, 33. doi: 10.1021/cr00099a003

(28) Bradley, D. C. Chem. Rev. 1989, 89, 1317. doi: 10.1021/ cr00096a004

(29) Brinker, C. J.; Scherer, G. W. Sol-Gel Science: the Physics and Chemistry of Sol-Gel Processing; Academic Press, Inc.: San Diego, CA, 1990.

(30) Ho, Y. D.; Chandra, M. B.; Lee, S. M.; Soo, C. Y. Sci. Rep. 2015, 5, 14353. doi: 10.1038/srep14353

(31) Hernandez-Borja, J.; Vorobiev, Y. V.; Ramirez-Bon, R. Sol. Energy Mater. Sol. Cells 2011, 95, 1882. doi: 10.1016/j. solmat.2011.02.012

(32) Obaid, A. S.; Hassan, Z.; Mahdi, M. A.; Bououdina, M. Sol. Energy 2013, 89, 143. doi: 10.1016/j.solener.2012.12.010

(33) Nicolau, Y. F. Appl. Surf. Sci. 1985, 22, 1061. doi: 10.1016/ 0378-5963(85)90241-7

(34) Ristov, M.; Sinadinovski G. J.; Grozdanov, I. Thin Solid Films 1985, 123, 63. doi: 10.1016/0040-6090(85)90041-0

(35) Pathan, H. M.; Lokhande, C. D. Bull. Mater. Sci. 2004, 27, 85. doi: 10.1007/BF02708491.
(36) Gao, Y.; Masuda, Y.; Yonezawa, A. T.; Koumoto, K. Chem. Mater. 2002, 14, 5006. doi: 10.1021/cm020358p

(37) Feng, L.; Liu, Y.; Hu, J. Langmuir 2004, 20, 1786. doi: $10.1021 / 1 \mathrm{a} 0357108$

(38) Jia, Q. X.; Mccleskey, T. M.; Burrell, A. K.; Lin, Y.; Collis, G. E.; Wang, H.; Li, A. D. Q.; Foltyn, S. R. Nat. Mater. 2004, 3, 329. doi: $10.1038 /$ nmat 1163

(39) Zou, G. F.; Luo, H. M.; Ronning, F.; Sun, B. Q.; McCleskey, T. M.; Burrell, A. K.; Bauer, E.; Jia, Q. X. Angew. Chem. Int. Ed. 2010, 49, 1782. doi: 10.1002/anie.200905804

(40) Lin, Y.; Wang, H.; Hawley, M. E.; Foltyn, S. R.; Jia, Q. X. Appl. Phys. Lett. 2004, 85, 3426. doi: 10.1063/1.1806265

(41) Jain, M.; Lin, Y.; Shukla, P.; Li, Y.; Wang, H.; Hundley, M. F.; Burrell, A. K.; McCleskey, T. M.; Foltyn, S. R.; Jia, Q. X. Thin Solid Films 2007, 515, 6411. doi: 10.1016/j.tsf.2006.11.144

(42) Liang, W. Z.; Ji, Y. D.; Nan, T.; Huang, J.; Bi, Z. X.; Zeng, H. Z.; Du, H.; Chen, C. L.; Jia, Q. X.; Lin, Y. ACS Appl. Mater. Interfaces 2012, 4, 2199. doi: 10.1021/am300205t

(43) Wimbush, S. C.; Li, M. C.; Vickers, M. E.; Maiorov, B.; Feldmann, D. M.; Jia, Q. X.; MacManus-Driscoll, J. L. Adv. Funct. Mater. 2009, 19, 835. doi: 10.1002/adfm.200801112

(44) Jain, M.; Bauer, E.; Ronning, F.; Hundley, M. F.; Civale, L.; Wang, H. Y.; Maiorov, B.; Burrell, A. K.; McClesky, T. M.; Foltyn, S. R.; DePaula, R. F.; Jia, Q. X. J. Am. Ceram. Soc. 2008, 91, 1858. doi: 10.1111/j.1551-2916.2008.02396.x

(45) Zou, G. F.; Luo, H. M.; Zhang, Y. Y.; Xiong, J.; Wei, Q. M.; Zhuo, M. J.; Zhai, J. Y.; Wang, H. Y.; Williams, D.; Li, N.; Bauer, E.; Zhang, X. H.; McCleskey, T. M.; Li, Y. R.; Burrell A. K.; Jia, Q. X. Chem. Commun. 2010, 46, 7837. doi: 10.1039/ C0CC01295E

(46) Zou, G. F.; Zhao, J.; Luo, H. M.; McCleskey, T. M.; Burrelld, A. K.; Jia, Q. X. Chem. Soc. Rev. 2014, 43, 2141. doi: 10.1039/ C3CS60285K

(47) Luo, H. M.; Lin, Y.; Wang, H. Y.; Lee, J. H.; Suvorova, N. A.; Mueller, A. H.; Burrell, A. K.; McCleskey, T. M.; Bauer, E.; Usov, I. O.; Hawley, M. E.; Holesinger, T. G.; Jia, Q. X. Adv. Mater. 2009, 21, 193. doi: 10.1002/adma.200801959

(48) Luo, H. M.; Wang, H. Y.; Zou, G. F.; Bauer, E.; McCleskey, T. M.; Burrell, A. K.; Jia, Q. X. Trans. Electr. Electron. Mater. 2010, 2, 54. doi: 10.4313/TEEM.2010.11.2.054

(49) Luo, H. M.; Zou, G. F.; Wang, H. Y.; Lee, J. H.; Lin, Y.; Peng, H. S.; Lin, Q. L.; Deng, S. G.; Bauer, E.; McCleskey, T. M.; Burrell, A. K.; Jia, Q. X. J. Phys. Chem. C 2011, 115, 17880. doi: 10.1021/jp2048376

(50) Li, X. L.; Thompson, J. D.; Zhang, Y. Y.; Brady, C. I.; Zou, G. F.; Mack, N. H.; Williams, D.; Duque, J. G.; Jia, Q. X.; Doorn, S. K. Nanoscale 2011, 3, 668. doi: 10.1039/C0NR00771D

(51) Zhang, Y. Y.; Sheehan, C. J.; Zhai, J. Y.; Zou, G. F.; Luo, H. M.; Xiong, J.; Zhu, Y. T.; Jia, Q. X. Adv. Mater. 2010, 22, 3027. doi: 10.1002/adma.200904426

(52) Zou, G. F.; Jain, M.; Yang, H.; Zhang, Y. Y.; Williams, D.; Jia, 
Q. X. Nanoscale 2010, 2, 418. doi: 10.1039/B9NR00257J

(53) Zou, G. F.; Yang, H.; Jain, M.; Zhou, H. H.; Williams, D.; Zhou, M.; McCleskey, T.; Burrell, A.; Jia, Q. X. Carbon 2009, 47, 933. doi: 10.1016/j.carbon.2008.11.017

(54) Ali, M. N.; Garcia1, M. A.; Parsons-Moss, T.; Nitsche, H. Nat. Protoc. 2010, 5, 1440. doi: 10.1038/nprot.2010.105

(55) McCleskey, T. M.; Shi, P.; Bauer, E.; Highland, M. J.; Eastman, J. A.; Bi, Z. X.; Fuoss, P. H.; Baldo, P. M.; Ren, W.; Scott, B. L.; Burrell, A. K.; Jia, Q. X. Chem. Soc. Rev. 2014, 43, 2141. doi: 10.1039/C3CS60285K

(56) Luo, H. M.; Jain, M.; McCleskey, T. M.; Bauer, E.; Burrell, A. K.; Jia, Q. X. Adv. Mater. 2007, 19, 3604. doi: 10.1002/ adma.200700528

(57) Lin, Y.; Xie, J.; Wang, H.; Li, Y.; Chavez, C.; Lee, S. Y.; Foltyn, S. R.; Crooker, S. A.; Burrell, A. K.; McCleskey, T. M.; Jia, Q. X. Thin Solid Films 2005, 492, 101. doi: 10.1016/j. tsf.2005.06.060

(58) Yi, Q. H.; Zhai, P. F.; Sun, Y. H.; Lou, Y. H.; Zhao, J.; Sun, B. Q.; Patterson, B.; Luo, H. M.; Zhang, W. R.; Jiao, L.; Wang, H. Y.; Zou, G. F. ACS Appl. Mater. Interfaces 2015, 7, 18218. doi: $10.1021 / \mathrm{am} 506367 \mathrm{~g}$

(59) Zhai, P. F.; Yi, Q. H.; Jian, J.; Wang, H. Y.; Song, P. Y.; Dong, C.; Lu, X.; Sun, Y. H.; Zhao, J.; Dai, X.; Lou, Y. H.; Yang, H.; Zou, G. F. Chem. Commun. 2014, 50, 1854. doi: 10.1039/ C3CC48877B

(60) Baber, S. M.; Lin, Q. L.; Zou, G. F.; Haberkorn, N.; Baily, S. A.; Wang, H. Y.; Bi, Z. X.; Yang, H.; Deng, S. G.; Hawley, M. E.; Civale, L.; Bauer, E.; McCleskey, T. M.; Burrell, A. K.; Jia, Q. X.; Luo, H. M. J. Phys. Chem. C 2011, 115, 25338. doi: $10.1021 /$ jp2068232

(61) Luo, H. M.; Jain, M.; Baily, S. A.; McCleskey, T. M.; Burrell, A. K.; Bauer, E.; DePaula, R. F.; Dowden, P. C.; Civale, L.; Jia, Q. X. J. Phys. Chem. B 2007, 111, 7497. doi: 10.1021/ jp0718451

(62) Du, J.; Gao, Y. F.; Luo, H. J,; Kang, L. T.; Zhang, Z. T.; Chen, Z.; Cao, C. X. Sol. Energy Mater. Sol. Cells 2011, 95, 469. doi: 10.1016/j.solmat.2010.08.035

(63) Rivadulla, F.; Bi, Z. X.; Bauer, E.; Rivas-Murias, B.; VilaFungueiriño, J. M.; Jia, Q. X. Chem. Mater. 2013, 25, 55. doi: 10.1016/j.solmat.2010.08.035

(64) Patta, Y. R.; Wesolowski, D. E.; Cima, M. J. Phys. C 2009, 469, 129. doi: 10.1016/j.physc.2008.12.006

(65) Lin, Y.; Lee, J. S.; Wang, H.; Li, Y.; Foltyn, S. R. Appl. Phys. Lett. 2004, 85, 5007. doi: 10.1063/1.1827927

(66) Cobas, R.; Muñoz-Perez, S.; Cadogan, J. M.; Puig, T.; Obradors, X. Appl. Phys. Lett. 2011, 99, 083113. doi: 10.1063/ 1.3629993

(67) Jain, M.; Shukla, P,; Li, Y,; Hundley, M. F.; Wang, H. Y.; Foltyn, S. R.; Burrell, A. K.; McCleskey, T. M.; Jia, Q. X. $A d v$. Mater. 2006, 18, 2695. doi: 10.1002/adma.200601221

(68) Alam, M. J.; Cameron, D. C. Thin Solid Films 2000, 377, 455. doi: 10.1016/S0040-6090(00)01369-9

(69) Guillén, C.; Herrero, J. Thin Solid Films 2005, 480, 129. doi: 10.1016/j.tsf.2004.11.040

(70) Chen, Z. X.; Li, W. C.; Li, R.; Zhang, Y. F.; Xua, G. Q.; Cheng, H. S. Langmuir 2013, 29, 45. doi: 10.1021/la4033282

(71) Liu, Y.; Lian, J. Appl. Surf. Sci. 2007, 253, 3727. doi: 10.1016/j. apsusc. 2006.08.012.

(72) Lee, H.W.; Lau, S. P.; Wang, Y. G.; Tse, K. Y.; Hng, H. H.; Tay, B. K. J. Crystal Growth 2004, 268, 596. doi: 10.1016/j. jcrysgro.2004.04.098

(73) Luo, L.; Bozyigit, D.; Wood, V.; Niederberger, M. Chem. Mater. 2013, 25, 4901. doi: 10.1021/cm4030149

(74) Peng, Q.; Kalanyan B.; Hoertz, P. G.; Miller, A.; Kim, D. H.; Hanson, K.; Leila, A.; Liu, J.; Meyer, T. J.; Parsons, G. N.; Glass, J. T. Nano Lett. 2013, 13, 1481. doi: 10.1021/n13045525.

(75) Ellmer, K. Nat. Photonics 2012, 6, 809. doi: 10.1038/ nphoton.2012.282

(76) Granqvist, C. G. Sol. Energy Mater. Sol. Cells 2007, 91, 1529. doi: 10.1016/j.solmat.2007.04.031

(77) Spaldin, N. A.; Fiebig, M. Science 2005, 309, 391. doi: 10.1126/science. 1113357

(78) Eerenstein, W.; Mathur, N. D.; Scott, J. F. Nature 2006, 442, 759. doi: 10.1038/nature05023.

(79) Cheong, S. W.; Mostovoy, M. Nat. Mater. 2007, 6, 13. doi: 10.1038/nmat1804

(80) Ramesh, R.; Spaldin, N. A. Nat. Mater. 2007, 6, 21. doi: $10.1038 /$ nmat 1805

(81) Luo, H. M.; Yang, H.; Baily, S. A.; Ugurlu, O.; Jain, M.; Hawley, M. E.; McCleskey, T. M.; Burrell, A. K.; Bauer, E.; Civale, L.; Holesinger, T. G.; Jia, Q. X. J. Am. Chem. Soc. 2007, 129, 14132. doi: 10.1021/ja075764u

(82) Luo, H. M.; Lin, Y.; Wang, H.; Chou, C. Y.; Suvorova, N. A.; Hawley, M. E.; Mueller, A. H.; Ronning, F.; Bauer, E.; Burrell, A. K.; McCleskey, T. M.; Jia, Q. X. J. Phys. Chem. C 2008, 112, 20535. doi: 10.1021/ja075764u

(83) Zou, G. F.; Jain, M.; Zhou, H. H.; Luo, H. M.; Baily, S. A.; Civale, L.; Bauer, E.; McCleskey, T. M.; Burrell, A. K.; Jia, Q. X. Chem. Commun. 2008, 45, 6022. doi: 10.1039/B815066D

(84) Luo, H. M.; Wang, H. Y.; Bi, Z. X.; Feldmann, D. M.; Wang, Y. Q.; Burrell, A. K. T.; McCleskey, M.; Bauer, E.; Hawley, M. E.; Jia, Q. X. J. Am. Chem. Soc. 2008, 130, 15224. doi: $10.1021 / \mathrm{ja} 803544 \mathrm{c}$

(85) Luo, H. M.; Wang, H. Y.; Bi, Z. X.; Zou, G. F.; McCleskey, T. M.; Burrell, A. K.; Bauer, E.; Hawley, M. E.; Wang, Y. Q.; Jia, Q. X. Angew. Chem. 2009, 121, 1518. doi: 10.1002/ anie. 200805394

(86) Halim, J.; Lukatskaya, M. R.; Cook, K. M.; Lu, J.; Smith, C. R.; Näslund, L. A.; May, S, J.; Hultman, L.; Gogotsi, Y.; Eklund, P.; Barsoum, M. W. Chem. Mater. 2014, 26, 2374. doi: $10.1021 / \mathrm{cm} 500641 \mathrm{a}$

(87) Xu, C.; Wang, L. B.; Liu, Z. B.; Chen, L.; Guo, J. K.; Kang, 
N.; Ma, X. L.; Cheng, H. M.; Ren, W. C. Nat. Mater. 2015, 14, 1135. doi: $10.1038 /$ nmat 4374

(88) Reyes, R.; Ni, C.; Bui, H. P.; Beebe, T. P.; Teplyakov, A. V. Chem. Mater. 2009, 21, 5163. doi: 10.1021/cm902107h

(89) Tanaka, S.; Kern, R. S.; Davis, R. F. Appl. Phys. Lett. 1994, 65, 2851. doi: 10.1063/1.112513.

(90) Phan, H. P.; Dao, D. V.; Wang, L.; Dinh, T.; Nguyen, N. T.; Qamar, A.; Tanner, P.; Dimitrijev, S.; Zhu, Y. J. Mater. Chem. C 2015, 3, 1172. doi: 10.1039/C4TC02679A

(91) Zou, G. F.; Wang, H. Y.; Mara, N.; Luo, H. M.; Li, N.; Di, Z. F.; Bauer, E.; Wang, Y. Q.; McCleskey, T.; Burrell, A.; Zhang, X. H.; Nastasi, M.; Jia, Q. X. J. Am. Chem. Soc. 2010, 132, 2516. doi: $10.1021 /$ ja9102315

(92) Wang, Y. J.; Wilkinson, D. P.; Zhang, J. J. Chem. Rev. 2011, 111, 7625. doi: 10.1021/cr100060r.

(93) Sahoo, N. G.; Pan, Y. Z.; Li, L.; Chan, S. H. Adv. Mater. 2012, 24, 4203. doi: 10.1002/adma.201104971

(94) Koenigsmann, C.; Wong, S. S. Energy Environ. Sci. 2011, 4, 1161. doi: 10.1039/C0EE00197J.

(95) Robert, C. L.; Vallé, K.; Pereira, F.; Sanchen, C. Chem. Soc. Rev. 2011, 40, 961. doi: 10.1039/c0cs00144a

(96) Kamat, P. V.; Tvrdy, K.; Baker, D. R.; Radich, J. G. Chem. Soc. Rev. 2010, 110, 6664. doi: 10.1021/cr100243p

(97) Gewirth, A. A.; Thorum, M. S. Inorg. Chem. 2010, 49, 3557. doi: $10.1021 /$ cr100243p
(98) Yang, W. B.; Duan, H. S.; Cha, K. C.; Hsu, C. J.; Hsu, W. C.; Zhou, H. P.; Bob, B.; Yang, Y. J. Am. Chem. Soc. 2013, 135, 6915. doi: 10.1021/ja312678c

(99) Scragg, J. J.; Kubart, T.; Wäjen, J. T.; Ericson, T.; Linnarsson, M. K.; Björkman, C. P. Chem. Mater. 2013, 25, 3162. doi: 10.1021/cm4015223

(100) Yang, W. B.; Duan, H. S.; Bob, B.; Zhou, H. P.; Lei, B.; Chung, C. H.; Li, S. H.; Hou, W. W.; Yang, Y. Adv. Mater. 2012, 24, 6323. doi: 10.1002/adma.201201785

(101) Zhang, Y. Y.; Zou, G. F.; Doorn, S. K.; Htoon, H.; Stan, L.; Hawley, M. E.; Sheehan, C. J.; Zhu, Y. T.; Jia, Q. X. ACS Nano 2009, 3, 2157. doi: 10.1021/nn9003988

(102) Yi, Q. H.; Dai, X.; Zhao, J.; Sun, Y. H.; Lou, Y. H.; Su, X. D.; Li, Q. W.; Sun, B. Q.; Zheng, H. H.; Shen, M. R.; Wang, Q. H.; Zou, G. F. Nanoscale 2013, 5, 6923. doi: 10.1039/ C3NR01857A

(103) Eda, G.; Chhowalla, M. Nano Lett. 2009, 9, 814.doi: 10.1021/ n18035367

(104) Zhang, Y. Y.; Ronning, F.; Gofryk, K.; Mara, N. A.; Haberkorn, N.; Zou, G. F.; Wang, H. Y.; Lee, J. H.; Bauer, E.; McCleskey, T. M.; Burell, A. K.; Civale, L.; Zhu, Y. T.; Jia, Q. X. Nanoscale 2012, 4, 2268. doi: 10.1039/c2nr11906d

(105) Wilke, R.; Bud'ko, S.; Canfield, P.; Finnemore, D.; Suplinskas, R.; Hannahs, S. Phys. Rev. Lett. 2004, 92, 217003. doi: 10.1103/PhysRevLett.92.217003 